\title{
Leading-term cycles of Harish-Chandra modules and partial orders on components of the Springer fiber
}

\author{
Peter E. Trapa
}

\begin{abstract}
We use the geometry of characteristic cycles of Harish-Chandra modules for a real semisimple Lie group $G_{\mathbb{R}}$ to prove an upper triangularity relationship between two bases of each special representation of a classical Weyl group. One basis consists of Goldie rank polynomials attached to primitive ideals in the enveloping algebra of the complexified Lie algebra $\mathfrak{g}$; the other consists of polynomials that measure the Euler characteristic of the restriction of an equivariant line bundle on the flag variety for $\mathfrak{g}$ to an irreducible component of the Springer fiber. While these two bases are defined only using the structure of the complex Lie algebra $\mathfrak{g}$, the relationship between them is closely tied to the real group $G_{\mathbb{R}}$. More precisely, the order leading to the upper triangularity result is a suborder of closure order for the orbits of the complexification of a maximal compact subgroup of $G_{\mathbb{R}}$ on the flag variety for $\mathfrak{g}$.
\end{abstract}

\section{Introduction}

The main result of this paper concerns a relationship between two natural bases of each special representation of a Weyl group. One basis arises in the study of primitive ideals in enveloping algebras, the other in the complex geometry of the Springer fiber. The relationship we uncover originates in the geometry of characteristic cycles of Harish-Chandra modules. As we detail below, it allows one to transport interesting information between the category of Harish-Chandra modules and the category of highest weight modules.

Before turning to applications, we formulate our main result in more detail. Let $\mathfrak{g}$ denote a complex semisimple Lie algebra, let $\mathfrak{B}$ denote its variety of Borel subalgebras, and fix a base point $\mathfrak{b}_{1}=\mathfrak{h}_{1} \oplus \mathfrak{n}_{1}$. (It would be more customary to call the base point simply $\mathfrak{b}$, but later $\mathfrak{b}$ will denote an arbitrary Borel subalgebra.) Let $W$ denote the Weyl group of $\mathfrak{h}_{1}$ in $\mathfrak{g}$, and write $\rho \in \mathfrak{h}_{1}^{*}$ for the halfsum of the roots of $\mathfrak{h}_{1}$ in $\mathfrak{b}_{1}$. Let $\mathcal{N}^{*}$ denote the nilpotent cone in $\mathfrak{g}^{*}$, and fix a complex nilpotent coadjoint orbit $\mathcal{O}$. Consider the set $\operatorname{Prim}_{\rho}(\mathfrak{g}, \mathcal{O})$ of two-sided ideals in the universal enveloping algebra $\mathrm{U}(\mathfrak{g})$ such that: (i) $I$ is the annihilator of a simple $\mathrm{U}(\mathfrak{g})$ module $X$ (i.e. $I$ is primitive); (ii) $I$ contains the augmentation ideal in the center of $\mathrm{U}(\mathfrak{g})$ (i.e. $I$ has infinitesimal character $\rho$ ); and (iii) with respect to the degree filtration, the associated graded ideal gr $I$ in the symmetric algebra $\mathrm{S}(\mathfrak{g})$ cuts out the closure $\overline{\mathcal{O}}$ of $\mathcal{O}$ (i.e. the associated variety of $I$ is $\overline{\mathcal{O}}$ ). This set is nonempty if and only if $\mathcal{O}$ is special [BV82, BV83]. To each primitive ideal $I$ in $\mathrm{U}(\mathfrak{g})$ (and hence to each element of $\left.\operatorname{Prim}_{\rho}(\mathfrak{g}, \mathcal{O})\right)$, Joseph attaches a harmonic homogeneous polynomial $p_{I} \in \mathrm{S}\left(\mathfrak{h}_{1}^{*}\right)$, the so-called Goldie rank polynomial [Jos80a, Jos80b]; see $\S 2.2$. Set

$$
\operatorname{Sp}(\mathcal{O}):=\operatorname{span}_{\mathbb{C}}\left\{p_{I} \mid I \in \operatorname{Prim}_{\rho}(\mathfrak{g}, \mathcal{O})\right\} .
$$

Received 15 October 2005, accepted in final form 2 August 2006.

2000 Mathematics Subject Classification 22E47.

Keywords: characteristic cycles, Harish-Chandra modules, Joseph polynomials.

The author was partially supported by NSF grant DMS-0300106.

This journal is (c) Foundation Compositio Mathematica 2007. 


\section{P. E. TRAPA}

Joseph proves that the elements $p_{I}$ are independent, and that the above span of this basis is an irreducible representation of $W$; it is called the special representation of $W$ parametrized by $\mathcal{O}$.

Now we introduce another basis of $\operatorname{Sp}(\mathcal{O})$. Let $T^{*} \mathfrak{B}$ denote the cotangent bundle to $\mathfrak{B}$, and write $\mu: T^{*} \mathfrak{B} \rightarrow \mathcal{N}^{*}$ for the moment map. Concretely we may write $T^{*} \mathfrak{B}=\left\{(\mathfrak{b}, \xi) \mid \xi \in(\mathfrak{g} / \mathfrak{b})^{*}\right\}$ and then $\mu$ maps a point $(\mathfrak{b}, \xi)$ to $\xi$ (or more precisely to the image of $\xi$ in $\mathfrak{g}^{*}$ under the canonical inclusion of $(\mathfrak{g} / \mathfrak{b})^{*}$ in $\left.\mathfrak{g}^{*}\right)$. For $\xi \in \mathcal{N}^{*}$, let $\mu^{-1}(\xi)$ denote the corresponding fiber, the so-called Springer fiber. Spaltenstein [Spa77] established that $\mu^{-1}(\xi)$ is equidimensional, and in particular that its irreducible components all have the same dimension. Write $\operatorname{Irr}\left(\mu^{-1}(\xi)\right)$ for the set of irreducible components. Now fix $\lambda \in \mathfrak{h}_{1}^{*}$ dominant and integral for the roots of $\mathfrak{h}_{1}$ in $\mathfrak{b}_{1}$, and let $\mathbb{C}_{\lambda}$ denote the corresponding one-dimensional representation of $B_{1}$. Consider the Borel-Weil line bundle $\mathcal{L}_{\lambda}:=G \times_{B_{1}} \mathbb{C}_{\lambda}$ and, for any subset $Z \subset \operatorname{Irr}\left(\mu^{-1}(\xi)\right)$, define $q_{Z}(\lambda)$ to be the Euler characteristic of $\mathcal{L}_{\lambda}$ restricted to $Z$. The assignment $\lambda \mapsto q_{Z}(\lambda)$ extends to a homogeneous polynomial $q_{Z} \in \mathrm{S}\left(\mathfrak{h}_{1}\right)$, sometimes called the Joseph polynomial after its introduction in a different context in [Jos84]; see also [Jos89] and the references therein, especially [Ros91]. The polynomials $q_{Z}$ are not independent for trivial reasons. The component group $A_{G}(\xi)$ of the centralizer of $\xi$ in $G$ clearly acts on $\operatorname{Irr}\left(\mu^{-1}(\xi)\right.$ ). (Here $G$ may be taken to be any complex connected Lie group with Lie algebra $\mathfrak{g}$; the action is insensitive to isogeny.) If $Z$ and $Z^{\prime}$ are actually irreducible components in the same $A_{G}(\xi)$ orbit, then $q_{Z}=q_{Z^{\prime}}$. In fact these are the only dependence relations, however, and the polynomials $q_{C}$ as $C$ ranges over $A_{G}(\xi) \backslash \operatorname{Irr}\left(\mu^{-1}(\xi)\right)$ are independent. Moreover, if we set $\mathcal{O}=G \cdot \xi$ and assume that this orbit is special, we have

$$
\operatorname{Sp}(\mathcal{O})=\operatorname{span}_{\mathbb{C}}\left\{q_{C} \mid C \in A_{G}(\xi) \backslash \operatorname{Irr}\left(\mu^{-1}(\xi)\right)\right\}
$$

with notation as in (1.1). Our main problem of interest, first posed by Joseph, is to relate the basis of the $q_{C}$ to the basis of the $p_{I}$.

Initially it was conjectured that (up to scaling) the two bases actually coincided [Jos84], and Melnikov proved that this is indeed the case for $\mathfrak{s l}(n, \mathbb{C})$ (see [Mel93]). Earlier Tanisaki provided examples (outside of Type A of course) where they did not coincide [Tan88]. He suggested the next possible alternative, namely that there is an upper triangular relation between the two. McGovern proved this in all classical cases [McG00]. Our main result is a proof of this result under a technical (conjecturally superfluous) hypothesis which we subsequently verify in many cases (including all classical types).

Theorem 1.2. Suppose $\mathcal{O}$ is a special nilpotent orbit in $\mathfrak{g}^{*}$ which satisfies Hypothesis $(\star)$. (This condition is described explicitly below. It holds if $\mathfrak{g}$ is classical and is conjectured to always hold; see $\S 5$.) Fix $\xi \in \mathcal{O}$. Then there is a bijection from

$$
A_{G}(\xi) \backslash \operatorname{Irr}\left(\mu^{-1}(\xi)\right) \longrightarrow \operatorname{Prim}_{\rho}(\mathfrak{g}, \mathcal{O})
$$

and a total order on these sets so that the matrix relating the $p$ - and $q$-bases is upper triangular. More precisely, use the total order to enumerate $A_{G}(\xi) \backslash \operatorname{Irr}\left(\mu^{-1}(\xi)\right)$ as $C_{1}, \ldots, C_{d}$ and let $I_{j}$ denote the image of $C_{j}$ under the above bijection. Then there are positive numbers $m_{i j}$ such that

$$
q_{I_{i}}=\sum_{j \leqslant i} m_{i j} p_{C_{j}}
$$

The main technique of the current paper interprets the bijection, the total order, and the coefficients using characteristic cycles of Harish-Chandra modules for real groups. (The more precise Theorem 3.13 makes this clear.) Once Hypothesis $(\star)$ is absorbed, the proofs rely only on a simple conceptual result of Chang [Cha93] which, roughly speaking, says that computing leadingterm cycles of Harish-Chandra modules is equivalent to relating the $p$ - and $q$-bases. Since there are (easy) triangularity results available for characteristic cycles, one obtains the current theorem. 


\section{LEADING-TERM CYCLES OF HARISH-CHANDRA MODULES}

As we remarked above, McGovern has established the conclusion of Theorem 1.2 for all classical cases (without assuming Hypothesis $(\star)$ ). However, our proof of Theorem 1.2 is very different from McGovern's: the total order he provides (which is essentially combinatorial) bears no obvious relation to ours (which is essentially geometric).

To discuss Hypothesis $(\star)$ we need some notation. Suppose $G_{\mathbb{R}}$ is a real linear reductive Lie group with Lie algebra $\mathfrak{g}_{\mathbb{R}}$. Let $K_{\mathbb{R}}$ denote its maximal compact subgroup, and write $K$ as the corresponding complexification. Let $\mathfrak{g}$ denote complexification of $\mathfrak{g}_{\mathbb{R}}$, and let $\mathfrak{g}=\mathfrak{k} \oplus \mathfrak{p}$ denote the complexified Cartan decomposition. $K$ acts on $\mathfrak{B}$ with a finite number of orbits. Given such an orbit, let $T_{Q}^{*} \mathfrak{B} \subset T^{*} \mathfrak{B}$ denote the conormal bundle to $Q$; concretely, it is easy to see that

$$
T_{Q}^{*} \mathfrak{B}=\left\{(\mathfrak{b}, \xi) \mid \mathfrak{b} \in Q \text { and } \xi \in(\mathfrak{g} / \mathfrak{b}+\mathfrak{k})^{*}\right\} .
$$

Thus, the moment map image $\mu\left(T_{Q}^{*} \mathfrak{B}\right)$ is a subvariety of the nilpotent cone elements in $(\mathfrak{g} / \mathfrak{k})^{*}$, which we denote as $\mathcal{N}_{\mathfrak{p}}^{*}$. (The notation reflects the fact that the Killing form identifies the nilpotent cone in $(\mathfrak{g} / \mathfrak{k})^{*}$ with the nilpotent cone in $\mathfrak{p}$.) Note that $\mu\left(T_{Q}^{*} \mathfrak{B}\right)$ is transparently invariant under the action of $K$, and, since $\mu$ is proper and $T_{Q}^{*} \mathfrak{B}$ is irreducible, $\mu\left(T_{Q}^{*} \mathfrak{B}\right)$ is irreducible as well. ${ }^{1}$ Since there are only a finite number of $K$ orbits on $\mathcal{N}_{\mathfrak{p}}^{*}$, it follows that there exists such an orbit, say $\mu(Q)$, such that $\mu(Q)$ is dense in $\mu\left(T_{Q}^{*} \mathfrak{B}\right)$. All such $K$ orbits on $\mathcal{N}_{\mathfrak{p}}^{*}$ arise in this way, and we partition $K \backslash \mathfrak{B}$ accordingly by defining, for $\mathcal{O}^{K} \in K \backslash \mathcal{N}_{\mathfrak{p}}^{*}$,

$$
\mu^{-1}\left(\mathcal{O}^{K}\right)=\left\{Q \in K \backslash \mathfrak{B} \mid \mu(Q)=\mathcal{O}^{K}\right\} .
$$

Recall that if $\mathcal{O}$ is a complex nilpotent orbit in $\mathfrak{g}^{*}$, then $\mathcal{O} \cap(\mathfrak{g} / \mathfrak{k})^{*}$ (if nonempty) is an equidimensional union of $K$ orbits [KR71], and of course all nilpotent $K$ orbits on $(\mathfrak{g} / \mathfrak{k})^{*}$ arise this way. We often implicitly assume that $\mathcal{O} \cap(\mathfrak{g} / \mathfrak{k})^{*}$ is nonempty and write $K \backslash\left(\mathcal{O} \cap(\mathfrak{g} / \mathfrak{k})^{*}\right)$ for the $K$ orbits on $\mathcal{O} \cap(\mathfrak{g} / \mathfrak{k})^{*}$.

We also need to recall some basic features of the geometry of the conormal bundle to an element of $\mu^{-1}\left(\mathcal{O}^{K}\right)$. Fix $\xi \in \mathcal{O}^{K}$, and define $A_{K}(\xi)$ to be the component group of the centralizer of $\xi$ in $K$. Because $K$ is a subgroup of $G, A_{K}(\xi)$ maps to $A_{G}(\xi)$, and so the orbits of $A_{G}(\xi)$ on $\operatorname{Irr}\left(\mu^{-1}(\xi)\right)$ break into potentially smaller $A_{K}(\xi)$ orbits. This certainly happens and presents some minor technical complications detailed in Definition 3.19 below. Now fix $Q \in \mu^{-1}\left(\mathcal{O}^{K}\right)$. According to Proposition 2.7 below, $T_{Q}^{*} \mathfrak{B} \cap \mu^{-1}(\xi)$ is dense in an $A_{K}(\xi)$ orbit, say $Z(Q)$, on $\operatorname{Irr}\left(\mu^{-1}(\xi)\right)$ and all such orbits may be written uniquely as $Z(Q)$ for $Q \in K \backslash \mathfrak{B}$.

Finally, we need to recall two representation-theoretic constructions. Given an irreducible HarishChandra module $X$ for $G_{\mathbb{R}}$, one may define an orbit $\operatorname{supp}_{\circ}(X) \in K \backslash \mathfrak{B}$ by considering the support of the appropriate localization of $X$ (Definition 3.1). By replacing $X$ by an associated graded object and passing to its support, one defines the associated variety of $X$; see $\S 3$. It transpires that $\operatorname{AV}(X)$ is a finite union of closures of $K$ orbits on $\mathcal{N}_{\mathfrak{p}}^{*}$. It follows easily from the definitions (see Proposition 3.10)

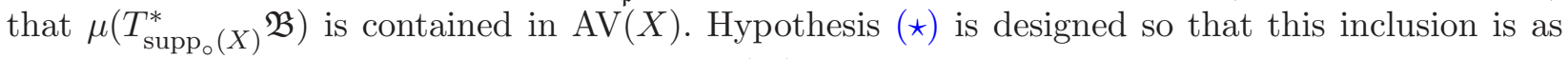
close to an equality as possible, given that $\operatorname{AV}(X)$ is generally reducible.

Hypothesis $(\star)$. Assume for the purposes of the introduction that the $A_{K}(\xi)$ orbits and $A_{G}(\xi)$ orbits on $\operatorname{Irr}\left(\mu^{-1}(\xi)\right)$ coincide; see $\S 3.3$ for the general case. A complex nilpotent orbit $\mathcal{O}$ for $\mathfrak{g}^{*}$ is said to satisfy Hypothesis $(\star)$ if is there is a real group $G_{\mathbb{R}}$ with complexified Lie algebra $\mathfrak{g}$ and an orbit $\mathcal{O}_{K}$ of $K$ on $\left(\mathcal{O} \cap(\mathfrak{g} / \mathfrak{k})^{*}\right)$ such that, for all $Q \in \mu^{-1}\left(\mathcal{O}_{K}\right)$, there exists an irreducible Harish-Chandra module $M_{Q}$ for $G_{\mathbb{R}}$ with trivial infinitesimal character with the properties that:

(i) $\operatorname{supp}_{\circ}\left(M_{Q}\right)=Q$; and

(ii) $\mu\left(T_{Q}^{*} \mathfrak{B}\right)$ is dense in an irreducible component of $\operatorname{AV}\left(M_{Q}\right)$.

${ }^{1}$ Actually, if $K$ is disconnected, then $T_{Q}^{*} \mathfrak{B}$ and hence $\mu\left(T_{Q}^{*} \mathfrak{B}\right)$ need not be irreducible. However, this complication is harmless and we ignore it for the purposes of the introduction. 


\section{P. E. TRAPA}

We conjecture that the more general Hypothesis $(\star)$ of $\S 3.3$ always holds for any special orbit $\mathcal{O}$. (By the above remarks, if Hypothesis $(\star)$ holds, $\mathcal{O}$ must indeed be special.) This is clearly a technical condition, but it does not seem inaccessible to general techniques. We have, however, been unable to prove it in general. Using less general techniques, we establish Hypothesis $(\star)$ for all special orbits for all classical types, for $\mathrm{G}_{2}$, and for some cases in $\mathrm{F}_{4}$; see $\S 5$. (As explained there, the Type D case is due to unpublished work of W. McGovern.)

In any event, we may now describe the bijection of Theorem 1.2. Retain the hypothesis of the theorem. In $\S 3$ (see Theorem 3.13), we prove that the map

$$
A_{G}(\xi) \cdot Z(Q) \mapsto \operatorname{Ann}\left(M_{Q}\right)
$$

is a bijection from

$$
A_{G}(\xi) \backslash \operatorname{Irr}\left(\mu^{-1}(\xi)\right) \rightarrow \operatorname{Prim}(\mathfrak{g}, \mathcal{O})_{\rho},
$$

as in the theorem. Since we have assumed (for the purposes of the introduction) that the $A_{K}(\xi)$ and $A_{G}(\xi)$ orbits coincide on $\operatorname{Irr}\left(\mu^{-1}(\xi)\right)$, the assignment $Q \in \mu^{-1}\left(\mathcal{O}_{K}\right)$ to $Z(Q) \in A_{G}(\xi) \backslash \operatorname{Irr}\left(\mu^{-1}(\xi)\right)$ described above is a bijection. Now $\mu^{-1}\left(\mathcal{O}_{K}\right)$ is a subset of $K$ orbits on $\mathfrak{B}$ and hence inherits the partial order arising from the closure order. Hence $A_{G}(\xi) \backslash \operatorname{Irr}\left(\mu^{-1}(\xi)\right)$ inherits this partial order and, according to the bijection in (1.3), so does $\operatorname{Prim}(\mathfrak{g}, \mathcal{O})_{\rho}$. Fix any total order compatible with this partial order. This total order and the bijection of (1.3) are the ones that appear in Theorem 1.2.

The construction of this bijection is interesting for several reasons. Most obviously, the problem of relating the $p$ - and $q$-bases seems like a problem that involves only the complex semisimple Lie algebra $\mathfrak{g}$, and yet the total order of the theorem is intimately related to the geometry of real groups. A second key observation is that, for a given complex orbit $\mathcal{O}$, there may be several real groups $G_{\mathbb{R}}$, or several $K$-orbits $\mathcal{O}_{K}$ for which Hypothesis $(\star)$ holds. In extreme cases, for instance, by using two different components $\mathcal{O}_{K}$ one can recover two total orders that are the exact opposite of each other; the immediate conclusion is that the matrix in this case is actually diagonal. (Understanding the family of partial orders obtained in this way on $A_{G}(\xi) \backslash \operatorname{Irr}\left(\mu^{-1}(\xi)\right)$ might be very enlightening.) In $\S 4$ we interpret these phenomena as a means to transfer information about characteristic cycles of Harish-Chandra modules between two different real forms of the same complex group; see Remark 3.14. In Remark 5.47, we indicate how this transfer could be related to information about the conjectural automorphic spectrum of a real group.

The theorem indicates the importance of Hypothesis $(\star)$. As remarked above, it holds for any special orbit $\mathcal{O}$ for a classical Lie algebra. Section 5 gives a complete status report, but for the purposes of the introduction we give one example. Suppose $\mathfrak{g}=\mathfrak{s p}(2 n, \mathbb{C})$ and fix $\mathcal{O}$. Then $\mathcal{O}$ is parametrized by a partition of $2 n$ in which all odd parts occur an even number of times. Suppose also that each even part $2 n_{i}$ occur an even number of times, say $2 d_{i}$. This latter condition is equivalent to the existence of some $p$ and $q$ with $p+q=n$ such that if $G_{\mathbb{R}}=\operatorname{Sp}(p, q)$, then $\mathcal{O} \cap(\mathfrak{g} / \mathfrak{k})^{*}$ is nonempty. In Theorem 5.2, we prove that Hypothesis $(\star)$ holds for $\mathcal{O}$ by choosing any irreducible component of $\mathcal{O} \cap(\mathfrak{g} / \mathfrak{k})^{*}$, and thus Theorem 1.2 applies. As we let $p$ and $q$ vary and the choice of irreducible component $\mathcal{O}_{K}$ varies, one obtains roughly $2^{\sum_{i} d_{i}}$ possibilities satisfying Hypothesis $(\star)$. The partial orders corresponding to each different choice are wildly different (although the bijections they define are the same). Piecing each of them together gives powerful information. See $\S 5$ for more details.

To conclude the introduction, it is worth detailing the content of our techniques in the special case of complex groups or, by the Bernstein-Gelfand-Gelfand-Enright-Joseph equivalence of categories (e.g. [BB85]), the highest weight category. Fix a Borel subalgebra $\mathfrak{b}_{1} \subset \mathfrak{g}$, write $B_{1}$ for the corresponding complex group, and let $L$ denote an irreducible $\left(\mathfrak{g}, B_{1}\right)$-module with trivial infinitesimal character. We may write $L=L(w \rho)$ in the standard parametrization, and recall the associated variety $\operatorname{AV}(L)$ (e.g. [Jos84]); see $\S 4$ below. Write $\overline{\mathcal{O}}=\operatorname{AV}(\operatorname{Ann}(L))$, and fix $\xi \in \mathcal{O}$. Then $\operatorname{AV}(L)$ is a union of the closures of a subset of the irreducible components $\mathbb{O}_{1}, \ldots, \mathbb{O}_{n}$ of $\mathcal{O} \cap\left(\mathfrak{g} / \mathfrak{b}_{1}\right)^{*}$. 


\section{LEADING-TERM CYCLES OF HARISH-CHANDRA MODULES}

Spaltenstein [Spa77] attached to each $\mathbb{O}_{i}$ an orbit $Z_{i}$ of $A_{G}(\xi)$ on $\operatorname{Irr}\left(\mu^{-1}(\xi)\right)$ (see Proposition 2.3). If we apply the equivalence of categories between $\left(\mathfrak{g}, B_{1}\right)$-modules and the category of HarishChandra bimodules for the complex group $G$, and use the properties of characteristic cycles developed in $\S 3$, we may conclude that

$$
\operatorname{AV}(L)=\overline{\mathbb{O}}_{1} \cup \cdots \cup \overline{\mathbb{O}}_{k} \quad \text { if and only if } \quad q_{\operatorname{Ann}\left(L\left(w^{-1}\right) \rho\right)}=\sum_{i-1}^{k} c_{i} p_{Z_{i}} \quad \text { with each } c_{i} \neq 0 .
$$

This (given the computations of [Hot84]) is the main result of [Jos84], and indeed the main motivation for his definition of the $p$-basis. (We sketch a simple proof of (1.4) using the techniques of this paper in Theorem 4.1 below.) Thus, Theorem 1.2 implies that the geometry of characteristic cycles of real groups has implications in the highest weight category. Earlier in [Tra05b, Theorem 1.3], using the computation of characteristic cycles of derived functor modules, we produced many new infinite families of highest weight modules with irreducible associated varieties, and different real forms gave different families. The present ideas may be seen as a significant extension.

\section{Background and notation}

\subsection{General notation}

Let $G$ be a complex reductive group. Let $G_{\mathbb{R}}$ be a real form of $G$ corresponding to a (complexified) Cartan involution $\theta$. Let $K_{\mathbb{R}}=G_{\mathbb{R}}^{\theta}$ denote the maximal compact subgroup of $G_{\mathbb{R}}$. Write $\mathfrak{g}_{\mathbb{R}}$ for the Lie algebra of $G_{\mathbb{R}}$, write $\mathfrak{g}$ for that of $G$, and likewise for $\mathfrak{k}_{\mathbb{R}}$ and $\mathfrak{k}$. Let $K$ be the corresponding complex subgroup of $G$ and write $\mathfrak{g}=\mathfrak{k} \oplus \mathfrak{p}$ for the Cartan decomposition with respect to $\theta$. Write $\mathcal{N}^{*}$ for the cone of nilpotents in $\mathfrak{g}^{*}$ and $\mathcal{N}_{\mathfrak{p}}^{*}$ for $\mathcal{N}^{*} \cap(\mathfrak{g} / \mathfrak{k})^{*}$. Let $\mathfrak{B}$ denote the variety of Borel subalgebras in $\mathfrak{g}$, and now revert to the more customary notation for a base point, $\mathfrak{b}=\mathfrak{h} \oplus \mathfrak{n}$, so that $\mathfrak{B}=G / B$. Let $\mu: T^{*} \mathfrak{B} \longrightarrow \mathcal{N}\left(\mathfrak{g}^{*}\right)$ denote the moment map for the $G$-action on $T^{*} \mathfrak{B}$. For $\xi \in \mathcal{N}^{*}$, write $\mu^{-1}(\xi)$ for the inverse image of $\xi$ under $\mu$. Let $W$ denote the Weyl group of $\mathfrak{h}$ in $\mathfrak{g}$.

Given a complex orbit $\mathcal{O}$, we write $K \backslash\left(\mathcal{O} \cap(\mathfrak{g} / \mathfrak{k})^{*}\right)$ for the set of $K$ orbits on $\mathcal{O} \cap(\mathfrak{g} / \mathfrak{k})^{*}$. When this notation is used, we often implicitly assume that $\mathcal{O} \cap(\mathfrak{g} / \mathfrak{k})^{*}$ is nonempty.

Given $\xi \in \mathcal{N}_{\mathfrak{p}}^{*}$, we let $A_{G}(\xi)$ denote the component group of $Z_{G}(\xi)$, the centralizer of $\xi$ in $G$, and write $A_{K}(\xi)$ for the component group of $Z_{K}(\xi)$. The groups $A_{G}(\xi)$ are sensitive to the isogeny class of $G$, but we will only study certain orbits of $A_{G}(\xi)$ which are insensitive to isogeny. (So, for instance, we could assume with little loss of generality that $G$ was adjoint.) In any event, the natural inclusion

$$
Z_{K}(\xi) \longrightarrow Z_{G}(\xi)
$$

descends to a map

$$
i_{\xi}: A_{K}(\xi) \longrightarrow A_{G}(\xi) .
$$

In general, $i_{\xi}$ is neither injective nor surjective. (First examples: surjectivity fails for the nonzero nilpotent orbit for $\operatorname{Sp}(1,1)$; injectivity fails for any orbit in $\operatorname{Sp}(4, \mathbb{R})$ with Jordan form $2^{2}$.) For an algebraic variety $Z$, we let $\operatorname{Irr}(Z)$ denote the set of its components. Note that $A_{G}(\xi)$ and $A_{K}(\xi)$ act on $\operatorname{Irr}\left(\mu^{-1}(\xi)\right)$, and, in particular, the orbits will coincide exactly when $i_{\xi}$ is surjective.

Given $Q \in K \backslash \mathfrak{B}$ and an irreducible $K$-equivariant local system $\psi$ supported on $Q$, we write $X(Q, \psi)$ for the Harish-Chandra module for $G_{\mathbb{R}}$ with trivial infinitesimal character corresponding to $\psi$ via localization as in [Mil93, Theorem H.5.3]. If $\psi=\mathbb{1}$, the trivial local system supported on $Q$, then we often write $X(Q)$ instead of $X(Q, \mathbb{1})$.

For $w \in W$, we write $L(w)$ for the simple highest weight module for $\mathfrak{g}$ with trivial infinitesimal character parameterized by $w$. We adopt the standard convention so that $L(e)$ is an irreducible Verma module, while $L\left(w_{\mathrm{o}}\right)$ is finite dimensional (where $w_{\mathrm{o}}$ is the long element of $W$ ). 


\section{P. E. TRAPA}

\subsection{Primitive ideals}

Let $\operatorname{Prim}(\mathrm{U}(\mathfrak{g}))$ denote the set of primitive ideals in $\mathrm{U}(\mathfrak{g})$. For each element $I \in \operatorname{Prim}(\mathrm{U}(\mathfrak{g}))$, let gr $I$ denote the ideal in $\mathrm{S}(\mathfrak{g})$ obtained from the grading of $I$ by degree in the enveloping algebra. Define $\operatorname{AV}(I)$, the associated variety of $I$, to be the subvariety of $\mathfrak{g}^{*}$ cut out by gr $I$. It is well known that $\operatorname{AV}(I)=\overline{\mathcal{O}}$ for some nilpotent orbit $\mathcal{O} \subset \mathcal{N}^{*}$ (see [BB82]).

It is easy to see that $I$ contains a unique maximal ideal of the center of $\mathrm{U}(\mathfrak{g})$. Write $\chi \in \mathfrak{h}^{*}$ for the dominant weight parameterizing this ideal via the Harish-Chandra isomorphism and write $I \in \operatorname{Prim}(\mathrm{U}(\mathfrak{g}))_{\chi}$. For any dominant integral $\lambda \in \mathfrak{h}^{*}$, write $I(\chi+\lambda) \in \operatorname{Prim}(\mathrm{U}(\mathfrak{g}))_{\chi+\lambda}$ for the primitive ideal obtained from $I$ using the translation functor from $\chi$ to $\chi+\lambda$. Finally, recall the Goldie rank polynomial $q_{I} \in S(\mathfrak{h})$ attached to $I$. This polynomial is characterized by the condition that its value at $\chi+\lambda$ is the Goldie rank of the primitive quotient $\mathrm{U}(\mathfrak{g}) / I(\chi+\lambda)$; see [Jos80a].

Primitive ideals with trivial infinitesimal character in Type B, C, or D are parametrized by standard domino tableaux of special shape, where the notion of specialness depends on the type [Gar90, Gar92, Gar93a].

\subsection{Fiber polynomials}

Fix $\xi \in \mathcal{N}\left(\mathfrak{g}^{*}\right)$ and $Z \subset \operatorname{Irr}\left(\mu^{-1}(\xi)\right)$. Fix a Borel subgroup $B$ and consider the function, $\phi_{Z}$ say, that assigns to each antidominant character $\chi$ of $B$ the Euler characteristic of the restriction of the Borel-Weil line bundle $G \times_{B} \mathbb{C}_{\chi}$ to $Z$. All cohomology groups are in fact finite, and thus $\phi_{Z}$ is an integer-valued function on the lattice of dominant weights in $\mathfrak{h}^{*}$. The following result may be extracted from [Jos89].

THEOREM 2.1. We have the following.

(i) The function $\phi_{Z}$ extends to a $W$-harmonic polynomial on $\mathfrak{h}^{*}$, which we denote $p_{Z}$.

(ii) If $C, C^{\prime} \in \operatorname{Irr}\left(\mu^{-1}(\xi)\right)$ are in the same $A_{G}(\xi)$ orbit, then $p_{C}=p_{C^{\prime}}$.

(iii) The relations in (ii) are the only dependence relations among the polynomials $\left\{p_{C} \mid C \in\right.$ $\left.\operatorname{Irr}\left(\mu^{-1}(\xi)\right)\right\}$. In particular, the polynomials $\left\{p_{Z} \mid Z \in A_{G}(\xi) \backslash \operatorname{Irr}\left(\mu^{-1}(\xi)\right)\right\}$ are independent. Moreover,

$$
\operatorname{Span}\left\{p_{Z} \mid Z \in A_{G}(\xi) \backslash \operatorname{Irr}\left(\mu^{-1}(\xi)\right)\right\}
$$

is $W$-invariant and isomorphic as a $W$-representation to $\operatorname{Sp}(G \cdot \xi)$, the representation attached to the trivial local system on $G \cdot \xi$ by the Springer correspondence.

Remark 2.2. Joseph originally defined the $p_{C}$ in a different way; again see [Jos89].

\subsection{Orbital varieties and the Springer fiber}

We recall the following result of Spaltenstein [Spa77]. In its statement, we fix a Borel subgroup $B$ whose nilradical has Lie algebra $\mathfrak{n}$, write $\eta$ for the projection of $G$ onto $G / B$, and write $\pi_{\xi}$ for the map $G \rightarrow G \cdot \xi$ defined by $g \mapsto g \cdot \xi$.

Proposition 2.3. Fix $\xi \in \mathcal{O} \in G \backslash \mathcal{N}\left(\mathfrak{g}^{*}\right)$. Then there is a bijection between $\operatorname{Irr}\left(\mathcal{O} \cap(\mathfrak{g} / \mathfrak{b})^{*}\right)$ and $A_{G}(\xi) \backslash \operatorname{Irr}\left(\mu^{-1}(\xi)\right)$. Given $Z$ in the latter set, $\pi_{\xi}\left(\eta^{-1}(Z)\right)$ meets a unique element $\mathbb{O}(Z)$ in the former set densely, and the bijection maps $Z$ to $\mathbb{O}(Z)$.

\subsection{Conormal bundles}

Given an orbit $Q \in K \backslash \mathfrak{B}$, write $T_{Q}^{*} \mathfrak{B}$ for the conormal bundle of $Q$ in $T^{*} \mathfrak{B}$. As above, let $\mu$ : $T^{*} \mathfrak{B} \longrightarrow \mathcal{N}\left(\mathfrak{g}^{*}\right)$ denote the moment map for the $G$-action on $T^{*} \mathfrak{B}$. As outlined in the introduction, the moment map image

$$
\mu\left(\overline{T_{Q}^{*} \mathfrak{B}}\right)
$$




\section{LEADING-TERM CYCLES OF HARISH-CHANDRA MODULES}

is always the closure of a single $K$ orbit on $\mathcal{N}_{\mathfrak{p}}^{*}$. To simplify notation as in the introduction,

$$
\text { we write } \mu(Q) \text { for the dense orbit of } K \text { in } \mu\left(\overline{T_{Q}^{*} \mathfrak{B}}\right) \text {. }
$$

For a given $\mathcal{O}_{K} \in K \backslash \mathcal{N}_{\mathfrak{p}}^{*}$, define

$$
\mu^{-1}\left(\mathcal{O}_{K}\right):=\left\{Q \in K \backslash \mathfrak{B} \mid \mu(Q)=\mathcal{O}_{K}\right\} .
$$

Fix any $\xi \in \mathcal{O}_{K}$, and recall the notation $A_{K}(\xi)$ for the component group of the centralizer of $\xi$ in $K$. (The following result is from a lecture by T. Springer.)

Proposition 2.7 (see, e.g., [Tra05a, Proposition 2.6.1]). Retain the above notation. Then

$$
\mu^{-1}(\xi) \cap T_{Q}^{*} \mathfrak{B}
$$

is dense in a unique orbit, say $Z(Q)$, of $A_{K}(\xi)$ on $\operatorname{Irr}\left(\mu^{-1}(\xi)\right)$. The map

$$
Q \longrightarrow Z(Q)
$$

is a bijection between $\mu^{-1}\left(\mathcal{O}_{K}\right)$ and $A_{K}(\xi) \backslash \operatorname{Irr}\left(\mu^{-1}(\xi)\right)$.

\subsection{Notation for fiber polynomials}

Given $Z \subset \operatorname{Irr}\left(\mu^{-1}(\xi)\right)$, recall the fiber polynomial $p_{Z}$ of $\S 2.3$. If $Q \in K \backslash \mathfrak{B}$, we write $p_{Q}$ for $p_{Z(Q)}$, where $Z(Q)=\mu^{-1}(\xi) \cap T_{Q}^{*} \mathfrak{B}$. If $\mathbb{O}$ is an irreducible component of $\mathcal{O} \cap(\mathfrak{g} / \mathfrak{b})^{*}$ and $Z(\mathbb{O}) \subset \operatorname{Irr}\left(\mu^{-1}(\xi)\right)$ is the subset corresponding to $\mathbb{O}$ by Proposition 2.3 , we write $p_{\mathbb{O}}$ for $p_{Z(\mathbb{O})}$.

\section{Characteristic cycles of Harish-Chandra modules}

We follow the notation of $\S 2.1$ and fix a real linear reductive group $G_{\mathbb{R}}$ with complexified Lie algebra $\mathfrak{g}$ and complexified Cartan decomposition $\mathfrak{g}=\mathfrak{k} \oplus \mathfrak{p}$. Let $\mathcal{D}$ denote the sheaf of algebraic differential operators on the flag variety $\mathfrak{B}$ of $\mathfrak{g}$.

Fix a finite length $(\mathfrak{g}, K)$ module $X$ with trivial infinitesimal character. Since the enveloping algebra $\mathrm{U}(\mathfrak{g})$ acts by global differential operators on $\mathfrak{B}$, the localization $\mathcal{X}=\mathcal{D} \otimes_{\mathrm{U}(\mathfrak{g})} X$ makes sense; it is a $\left(\mathcal{D}_{\mathfrak{B}}, K\right)$ module.

Definition 3.1. The support of a $(\mathfrak{g}, K)$ module $X$ with trivial infinitesimal character is defined to be the support of the localization $\mathcal{X}$. This is a $K$ invariant subvariety of $\mathfrak{B}$. If $X$ is irreducible, then the support of $X$ is the closure of a unique $K$ orbit on $X$ (e.g. [Mil93, Lemma H.5.1]). We denote this orbit by $\operatorname{supp}_{\circ}(X)$.

\subsection{Characteristic varieties}

We need a microlocalization of the support construction. Choose a good $K$ invariant filtration $\mathcal{X}^{j}$ of $\mathcal{X}$ compatible with the degree filtration on $\mathcal{D}$ and pass to the associated graded object gr $\mathcal{X}$. This is a $(\operatorname{gr} \mathcal{D}, K)$ module. Since the symbol calculus identifies $\operatorname{gr} \mathcal{D}$ with functions on $T^{*} \mathfrak{B}$, we may view gr $\mathcal{X}$ as an $\left(\mathbb{O}_{T^{*} \mathfrak{B}}, K\right)$ module, where $\mathbb{O}_{T^{*} \mathfrak{B}}$ denotes the structure sheaf of $T^{*} \mathfrak{B}$. Since $X$ was assumed to have finite length (and hence to be finitely generated), it follows that we may identify gr $\mathcal{X}$ with a $K$-equivariant coherent sheaf on $T^{*} \mathfrak{B}$. Define the characteristic variety of $X$, denoted by $\mathrm{CV}(X)$, to be the support of the sheaf gr $\mathcal{X}$. This is transparently a $K$-equivariant subvariety of $T^{*} \mathfrak{B}$, but since $\mathcal{X}$ is a special kind of $\mathcal{D}$ module (arising as the localization of $X$ ), much more is true: there exists a subset $\operatorname{cv}(X)$ of $K$ orbits on $\mathfrak{B}$ such that

$$
\mathrm{CV}(X)=\bigcup_{Q \in \operatorname{cv}(X)} \overline{T_{Q}^{*} \mathfrak{B}}
$$




\section{P. E. TRAPA}

here $\operatorname{cv}(X)$ is a subset of $K \backslash X$. See [Mil93, Proposition H.3.6] for more details. It is also useful to keep track of the rank of the sheaf gr $X$ along each irreducible component. The resulting invariant is called the characteristic cycle and is denoted by

$$
\mathcal{C V}(X)=\sum_{Q \in \operatorname{cv}(X)} m_{Q} \overline{T_{Q}^{*} \mathfrak{B}}
$$

here each $m_{Q}$ is a positive integer. Both invariants are independent of the filtration initially chosen. Clearly each construction is additive on short exact sequences and descends to the appropriate Grothendieck group.

There is no effective algorithm known to compute $\operatorname{cv}(X)$. The following result provides some very weak information; its proof is quite easy and follows from the considerations around [Mil93, Proposition H.3.6], for instance.

Proposition 3.2. Let $X$ be the Harish-Chandra module with trivial infinitesimal character. Recall its support (Definition 3.1). Then

$$
\operatorname{supp}_{\circ}(X) \in \operatorname{cv}(X) \text { and } m_{\text {supp }_{\circ}}(X)=1
$$

and

$$
\operatorname{cv}(X) \subset\left\{Q \in K \backslash \mathfrak{B} \mid Q \subset \overline{\operatorname{supp}_{\circ}(X)}\right\} .
$$

Example 3.5. There is one important case where the computation of characteristic cycles is easy. Suppose $Q \in K \backslash \mathfrak{B}$ is closed. Recall the notation $X(Q)=X(Q, \mathbb{1})$ of $\S 2.1$. Then (3.3) and (3.4) taken together imply that

$$
\mathcal{C V}(X(Q))=1 \cdot\left[\overline{T_{Q}^{*} \mathfrak{B}}\right]
$$

We may pursue this argument more generally by localizing on a partial flag variety. More precisely, suppose there exists a $\theta$-stable parabolic $\mathfrak{s}$ of $\mathfrak{g}$. (The notation $\mathfrak{q}$ would be more customary, but the letter 'q' already has been taken.) Write $S$ for the corresponding subgroup of $G$. Fix a Borel subalgebra $\mathfrak{b} \subset \mathfrak{s}$ and write $B$ for the corresponding subgroup of $S$. Write $\pi$ for the projection from $G / B$ to $G / S$. Suppose $Q \in K \backslash \mathfrak{B}$ is dense in the preimage of the (closed) orbit of $\mathfrak{s}$ under $K$ on $G / S$. Then $X(Q)$ is a derived functor module of the form $A_{\mathfrak{s}}$ (in the terminology of [VZ84]). It follows that

$$
\mathcal{C V}(X(Q))=1 \cdot\left[\overline{T_{Q}^{*} \mathfrak{B}}\right]
$$

which generalizes (3.6). This will be important in Example 3.21 below.

We have assumed that $X$ has trivial infinitesimal character. We could have just as easily worked with any infinitesimal character that differed from the trivial one by a weight of a finite-dimensional representation of $G$. In this setting, we localize using a sheaf of twisted differential operators on the flag variety, and define $\mathcal{C V}(X)$ just as above.

Proposition 3.8. Assume for simplicity that $G_{\mathbb{R}}$ is connected. Suppose $X$ is an irreducible HarishChandra module with trivial infinitesimal character. Choose a representative $\rho$ of the infinitesimal character, or equivalently a system of positive roots $\Delta^{+}$for a fixed Cartan $\mathfrak{h}$ in $\mathfrak{g}$. Suppose $\nu$ is the highest weight of a finite-dimensional representation of $G$ that is dominant for $\Delta^{+}$. Define the translation functor $\psi_{\rho}^{\rho+\nu}$ from infinitesimal character $\rho$ to $\nu+\rho$ (e.g. [KV95, Equation (7.141)]) and set $X(\nu)=\psi_{\rho}^{\rho+\nu}(X)$. Then

$$
\mathcal{C V}(X)=\mathcal{C V}(X(\nu))
$$

(If $G_{\mathbb{R}}$ is disconnected, the extra technical requirements needed to define translation functors are treated in [KV95, Theorem 2.229]. Once the is done, the conclusion of (3.9) holds.) 


\section{LEADING-TERM CYCLES OF HARISH-CHANDRA MODULES}

\subsection{Associated varieties}

The construction of the characteristic variety may be imitated without localizing; see [Vog91]. One chooses a good $K$-invariant filtration $X^{j}$ on $X$ compatible with the degree filtration on the enveloping algebra. The associated graded $\operatorname{gr} X$ is a $(\operatorname{gr} \mathrm{U}(\mathfrak{g}), K)$ module. Of course $\operatorname{gr} \mathrm{U}(\mathfrak{g})=$ $\mathrm{S}(\mathfrak{g})$, and, since the filtration was chosen to be $K$-invariant, the action of $\mathrm{S}(\mathfrak{g})$ factors to $\mathrm{S}(\mathfrak{g} / \mathfrak{k})$. Thus, gr $X$ is a finitely generated $(\mathrm{S}(\mathfrak{g} / \mathfrak{k}), K)$ module, i.e. a $K$-equivariant coherent sheaf on $(\mathfrak{g} / \mathfrak{k})^{*}$. The associated variety of $X$, denoted by $\operatorname{AV}(X)$, is defined to be the support of this sheaf. It is a $K$-invariant subvariety of $(\mathfrak{g} / \mathfrak{k})^{*}$, but again since $X$ is a special kind of $\mathrm{U}(\mathfrak{g})$ module (annihilated by a central ideal of finite codimension) much more is true: there exists a subset av $(X)$ of $K$ orbits on $\mathcal{N}(\mathfrak{g} / \mathfrak{k})^{*}$ such that

$$
\operatorname{AV}(X)=\bigcup_{\mathcal{O}_{K} \in \operatorname{av}(X)} \overline{\mathcal{O}_{K}}
$$

In fact, $\mathcal{O}:=G \cdot \mathcal{O}_{K}$ is well defined independent of the choice of $\mathcal{O}_{K} \in \operatorname{av}(X)$ and, moreover, $\mathcal{O}$ is dense in $\operatorname{AV}(\operatorname{Ann}(X))$. According to a theorem of Barbasch and Vogan [BV82], $\mathcal{O}$ is a special orbit. Again we may keep track of the rank of the sheaf gr $X$ along each irreducible component and define the associated cycle

$$
\mathcal{A V}(X)=\sum_{\mathcal{O}_{K} \in \operatorname{av}(X)} m_{\mathcal{O}_{K}} \overline{\mathcal{O}_{K}}
$$

where each $m_{\mathcal{O}_{K}}$ is a positive integer. Again both $\operatorname{AV}(X)$ and $\mathcal{A} \mathcal{V}(X)$ do not depend on the choice of filtration.

There is a simple relationship between the sets $\operatorname{cv}(X)$ and $\operatorname{av}(X)$.

Proposition 3.10. Let $X$ be a finite-length $(\mathfrak{g}, K)$ module with trivial infinitesimal character. Let $\mu$ denote the moment map $T^{*} \mathfrak{B} \rightarrow \mathfrak{g}^{*}$ and recall the notation of (2.4). Then $\mu(\mathrm{CV}(X))=\operatorname{AV}(X)$; that is,

$$
\operatorname{av}(X)=\{\mu(Q) \mid Q \in \mathrm{cv}(X)\} .
$$

Consequently, in the notation of Propositions 3.2 and 3.8,

$$
\operatorname{AV}(X)=\operatorname{AV}(X(\nu)) .
$$

The relationship between the multiplicities in $\mathcal{C} \mathcal{V}(X)$ and $\mathcal{A} \mathcal{V}(X)$ is more subtle, however, as must be the case by the following result.

Proposition 3.11 (See, e.g., the proof of [Vog78, Corollary 4.4]). Retain the notation of Proposition 3.8. Fix $\mathcal{O}_{K} \in \operatorname{av}(X)$. By Proposition 3.10, $\mathcal{O}_{K} \in \operatorname{av}(X(\nu))$, and so we may consider the assignment

$$
\nu \mapsto m_{\mathcal{O}_{K}}(\nu):=m_{\mathcal{O}_{K}}(X(\nu))
$$

Then $m_{\mathcal{O}_{K}}$ extends to a harmonic polynomial on $\mathfrak{h}$.

Roughly speaking, for suitably compatible choices of filtrations, gr $X$ is the pushforward of gr $\mathcal{X}$ via the moment map $\mu$. So to compute the rank of gr $X$ along an irreducible component, we have to compute the integral over the fiber of $\mu$, weighted by the appropriate rank of gr $\mathcal{X}$. To make that precise we need a little more notation. Given $\mathcal{O}_{K} \in \mathrm{av}(X)$, define (using the notation of (2.6) and $(2.5))$

$$
\operatorname{cv}\left(X ; \mathcal{O}_{K}\right):=\operatorname{cv}(X) \cap \mu^{-1}\left(\mathcal{O}_{K}\right)=\left\{Q \in \operatorname{cv}(X) \mid \mu(Q)=\mathcal{O}_{K}\right\},
$$

and write

$$
\mathrm{CV}\left(X ; \mathcal{O}_{K}\right)=\bigcup_{Q \in \operatorname{cv}\left(X ; \mathcal{O}_{K}\right)} \overline{T_{Q}^{*} \mathfrak{B}}
$$




\section{P. E. TRAPA}

and

$$
\mathcal{C V}\left(X ; \mathcal{O}_{K}\right)=\sum_{Q \in \operatorname{cv}\left(X ; \mathcal{O}_{K}\right)} m_{Q} \overline{T_{Q}^{*} \mathfrak{B}}
$$

We call this the leading term of the characteristic cycle of $X$ over $\mathcal{O}_{K}$. The pushforward argument may be quantified precisely as follows.

Proposition 3.12 (See [Cha93, Corollary 2.5.6]). Retain the notation of Proposition 3.8, and fix any $\mathcal{O}_{K} \in \operatorname{av}(X)=\operatorname{av}(X(\nu))$. Then

$$
m_{\mathcal{O}_{K}}(\nu)=\sum_{Q \in \operatorname{cv}\left(X ; \mathcal{O}_{K}\right)} m_{Q} p_{Q}(\nu)
$$

with the notation as in $\S 2.6$.

An argument reproduced in [Cha93, § 1.6] shows that the multiplicity polynomial in Proposition 3.12 is in fact proportional to the Goldie rank polynomial of the annihilator of $X$. More precisely, we have the following result.

Theorem 3.13. Suppose $X$ has integral infinitesimal character. Fix any $\mathcal{O}_{K} \in$ av $(X)$. Then there is a nonzero constant $c$ such that

$$
q_{\mathrm{Ann}(X)}=c \sum_{Q \in \mathrm{cv}\left(X ; \mathcal{O}_{K}\right)} m_{Q} p_{Q}
$$

with the notation as in $\S \S 2.2$ and 2.6.

Remark 3.14. It is important to note that the theorem holds for any $\mathcal{O}_{K} \in \operatorname{av}(X)$. Recall (from Theorem 2.1) that the polynomials $p_{Q}$ are nearly independent. Thus, Theorem 3.13 says (up to the slight potential dependence of the $\left.p_{Q}\right)$ that once one computes $\mathcal{C V}\left(X ; \mathcal{O}_{K}\right)$ for some $\mathcal{O}_{K} \in \operatorname{av}(X)$, then one may transfer this computation to compute $\mathcal{C V}\left(X ; \mathcal{O}_{K}^{\prime}\right)$ for any other $\mathcal{O}_{K}^{\prime} \in \operatorname{av}(X)$.

However, even more is true: since the expression of Goldie rank polynomials in terms of fiber polynomials does not depend on the real form (apart from the minor complication posed by the difference between the $A_{K}(\xi)$ and $A_{G}(\xi)$ orbits on $\operatorname{Irr}\left(\mu^{-1}(\xi)\right)$ ), one may transfer the computation of leading-term cycles between two different real forms of the same complex group. This should have interesting and possibly deep applications. Some are proposed in Remark 5.47.

Theorem 3.13 has additional significant philosophical import. It says that the information of the annihilator of $X$ is encoded in the leading term of the characteristic cycle of $X$ over any irreducible component of $\mathrm{AV}(X)$. This gives a geometric interpretation of the annihilator of a Harish-Chandra module. (Such ideas were implicitly present in the context of Harish-Chandra bimodules in [BB85].)

We have assembled the set of tools to prove Theorem 1.2 in the case when the orbits of $A_{K}(\xi)$ and $A_{G}(\xi)$ coincide. In fact, we have the following more precise result.

Theorem 3.15. Fix a special orbit $\mathcal{O}$ and $\mathcal{O}_{K} \in K \backslash\left(\mathcal{O} \cap(\mathfrak{g} / \mathfrak{k})^{*}\right)$. Assume the orbits of $A_{K}(\xi)$ and $A_{G}(\xi)$ on $\operatorname{Irr}\left(\mu^{-1}(\xi)\right)$ coincide. Suppose that Hypothesis $(\star)$ (described in the introduction) holds for $\mathcal{O}_{K}$. Enumerate $\mu^{-1}\left(\mathcal{O}_{K}\right)$ as $Q_{1}, \ldots, Q_{d}$ and the Harish-Chandra modules in Hypothesis $(\star)$ as $M_{Q_{1}}, \ldots, M_{Q_{d}}$.

(i) Recall the notation of Proposition 2.7; so $\mu^{-1}(\xi) \cap T_{Q_{i}}^{*} \mathfrak{B}$ is dense in $Z\left(Q_{i}\right)$. The map

$$
\operatorname{Ann}\left(M_{Q_{i}}\right) \longmapsto Z\left(Q_{i}\right)
$$

defines a bijection

$$
\operatorname{Prim}(\mathfrak{g}, \mathcal{O})_{\rho}:=\left\{I \in \operatorname{Prim}(\mathrm{U}(\mathfrak{g}))_{\rho} \mid \operatorname{AV}(I)=\overline{\mathcal{O}}\right\} \longrightarrow A_{G}(\xi) \backslash \operatorname{Irr}\left(\mu^{-1}(\xi)\right) .
$$

In particular, the primitive ideals $\operatorname{Ann}\left(M_{Q_{i}}\right)$ are distinct. 


\section{LEADING-TERM CYCLES OF HARISH-CHANDRA MODULES}

(ii) Choose any total order on $\mu^{-1}\left(\mathcal{O}_{K}\right) \subset K \backslash \mathfrak{B}$ compatible with the closure order on $K \backslash \mathfrak{B}$, and reorder indices so that $i<j$ implies $Q_{i}<Q_{j}$ in this total order. Set $I_{i}=\operatorname{Ann}\left(M_{Q_{i}}\right)$, let $d=\operatorname{dim}(\operatorname{Sp}(\mathcal{O}))$, and define a $d \times d$ matrix $\left(M_{i j}\right)$ by

$$
q_{I_{j}}=\sum_{i} m_{i j} p_{Q_{i}}
$$

Then $M$ is upper triangular.

Proof. Define the matrix $M$ as indicated in part (ii) of Theorem 3.15. Theorem 3.13 together with Proposition 3.2 imply that the matrix is upper triangular with nonzero entries on the diagonal. (The fact that the matrix is triangular follows from (3.4); the fact that the diagonal entries are nonzero follows from (3.3).) This proves Theorem 3.15(ii). It also proves the fact that the Goldie rank polynomials of the various primitive ideals $I_{j}$ are independent. Hence, the various primitive ideals $I_{j}$ are distinct. This is the last assertion of Theorem 3.15(i). The theory of Goldie rank polynomials mentioned in the introduction implies that cardinality of $\operatorname{Prim}(\mathfrak{g}, \mathcal{O})_{\rho}$ is $d$, and so the various $I_{j}$ exhaust $\operatorname{Prim}(\mathfrak{g}, \mathcal{O})_{\rho}$. Meanwhile, the number of $A_{G}(\xi)$ orbits on $\operatorname{Irr}\left(\mu^{-1}(\xi)\right)$ is $d$ by Theorem 2.1, and so the two sets in (3.16) have the same cardinality. Finally, the map of Theorem 3.15(i) is injective by Proposition 2.7. Hence, it is bijective and Theorem 3.15 is proved.

\subsection{Hypothesis $(\star)$ and Theorem 1.2 in general}

Theorem 3.15 and the discussion in the introduction assumed that the $A_{G}(\xi)$ and $A_{K}(\xi)$ orbits on $\operatorname{Irr}\left(\mu^{-1}(\xi)\right)$ coincided. In this section, we treat the mild complications that present themselves if that is not the case.

Fix $Q \in \mu^{-1}\left(\mathcal{O}_{K}\right)$ (with the notation as in (2.6)) and $\xi \in \mathcal{O}_{K}$. Recall from Proposition 2.7 that $T_{Q}^{*} \mathfrak{B} \cap \mu^{-1}(\xi)$ is dense in an $A_{K}(\xi)$ orbit $Z(Q)$ on $\operatorname{Irr}\left(\mu^{-1}(\xi)\right)$. The map

$$
\begin{aligned}
\Phi: \mu^{-1}\left(\mathcal{O}_{K}\right) & \longrightarrow A_{G}(\xi) \backslash \operatorname{Irr}\left(\mu^{-1}(\xi)\right) \\
Q & \longrightarrow A_{G}(\xi) \cdot Z(Q)
\end{aligned}
$$

is surjective by Proposition 2.7 and the fact that each $A_{G}(\xi)$ orbit is a union of $A_{K}(\xi)$ orbits. Recall that $\mu^{-1}(\mathcal{O})$ is partially ordered by the closure order on $K \backslash \mathfrak{B}$. The following is the definition we need.

Definition 3.19. A subset $\mu^{-1}\left(\mathcal{O}^{K}\right)^{\prime} \subset \mu^{-1}\left(\mathcal{O}^{K}\right)$ is said to be of minimal type if the map $\Phi$ of (3.17) restricts to a bijection

$$
\begin{aligned}
\mu^{-1}\left(\mathcal{O}_{K}\right)^{\prime} & \longrightarrow A_{G}(\xi) \backslash \operatorname{Irr}\left(\mu^{-1}(\xi)\right) \\
Q & \longrightarrow A_{G}(\xi) \cdot Z(Q)
\end{aligned}
$$

and if, furthermore, whenever $Q^{\prime} \in \mu^{-1}\left(\mathcal{O}_{K}\right)^{\prime}$ and $Q \in \mu^{-1}\left(\mathcal{O}_{K}\right)$ are such that $\Phi(Q)=\Phi\left(Q^{\prime}\right)$, then $Q^{\prime} \subset \bar{Q}$. Hence, a subset $\mu^{-1}\left(\mathcal{O}_{K}\right)^{\prime}$ of minimal type is constructed by picking an element $Q^{\prime}$ out of each fiber of $\Phi$ with the property that $Q^{\prime}$ is minimal in the closure order restricted to the fiber. Note that the cardinality of any subset of minimal type is simply that of $A_{G}(\xi) \backslash \operatorname{Irr}\left(\mu^{-1}(\xi)\right)$, the dimension of $\operatorname{Sp}(\mathcal{O})$. Note also that it is obvious that if the $A_{G}(\xi)$ and $A_{K}(\xi)$ orbits on $\operatorname{Irr}\left(\mu^{-1}(\xi)\right)$ coincide, then the only subset of $\mu^{-1}\left(\mathcal{O}^{K}\right)$ which is of minimal type is $\mu^{-1}\left(\mathcal{O}^{K}\right)$ itself.

The following is the general version of Hypothesis $(\star)$; when the orbits of $A_{K}(\xi)$ and $A_{G}(\xi)$ coincide it restricts to the version given in the introduction.

Hypothesis $(\star)$. A complex nilpotent orbit $\mathcal{O}$ for $\mathfrak{g}^{*}$ is said to satisfy Hypothesis $(\star)$ if there is a real group $G_{\mathbb{R}}$ with complexified Lie algebra $\mathfrak{g}$, an irreducible component $\mathcal{O}_{K}$ of $K \backslash\left(\mathcal{O} \cap(\mathfrak{g} / \mathfrak{k})^{*}\right)$, and a subset $\mu^{-1}\left(\mathcal{O}_{K}\right)^{\prime}$ of $\mu^{-1}\left(\mathcal{O}_{K}\right)$ of minimal type (Definition 3.19), such that, for all $Q \in \mu^{-1}\left(\mathcal{O}_{K}\right)^{\prime}$, 


\section{P. E. TRAPA}

there exists a Harish-Chandra module $M_{Q}$ for $G_{\mathbb{R}}$ with trivial infinitesimal character with the properties that:

(i) $\operatorname{supp}_{\circ}\left(M_{Q}\right)=Q$; and

(ii) $\mu\left(T_{Q}^{*} \mathfrak{B}\right)$ is dense in an irreducible component of $\operatorname{AV}\left(M_{Q}\right)$.

The following is the more general version of Theorem 3.15. Its proof follows in exactly the same way.

Theorem 3.20. Fix a special orbit $\mathcal{O}$ and $\mathcal{O}_{K} \in K \backslash\left(\mathcal{O} \cap(\mathfrak{g} / \mathfrak{k})^{*}\right)$. Suppose that Hypothesis $(\star)$ holds for $\mathcal{O}_{K}$ and the subset $\mu^{-1}\left(\mathcal{O}_{K}\right)^{\prime}$ of minimal type. Enumerate $\mu^{-1}\left(\mathcal{O}_{K}\right)^{\prime}$ as $Q_{1}, \ldots, Q_{d}$ and the Harish-Chandra modules in Hypothesis ( $\star$ ) as $M_{Q_{1}}, \ldots, M_{Q_{d}}$.

(i) Then the map

defines a bijection

$$
\operatorname{Ann}\left(M_{Q_{i}}\right) \longmapsto \mu^{-1}(\xi) \cap T_{Q_{i}}^{*} \mathfrak{B}
$$

$$
\operatorname{Prim}(\mathfrak{g}, \mathcal{O})_{\rho}:=\left\{I \in \operatorname{Prim}(\mathrm{U}(\mathfrak{g}))_{\rho} \mid \operatorname{AV}(I)=\overline{\mathcal{O}}\right\} \longrightarrow A_{G}(\xi) \backslash \operatorname{Irr}\left(\mu^{-1}(\xi)\right) .
$$

In particular, the primitive ideals $\operatorname{Ann}\left(M_{Q_{i}}\right)$ are distinct.

(ii) Choose any total order on $\mu^{-1}\left(\mathcal{O}_{K}\right)^{\prime} \subset K \backslash \mathfrak{B}$ compatible with the closure order on $K \backslash \mathfrak{B}$, and reorder indices so that $i<j$ implies $Q_{i}<Q_{j}$ in this total order. Set $I_{i}=\operatorname{Ann}\left(M_{Q_{i}}\right)$, let $d=\operatorname{dim}(\operatorname{Sp}(\mathcal{O}))$, and define a $d \times d$ matrix $\left(M_{i j}\right)$ by

$$
q_{I_{j}}=\sum_{i} m_{i j} p_{Q_{i}} .
$$

Then $M$ is upper triangular.

Example 3.21. Recall Example 3.5 above. Fix $\mathcal{O}^{K}$ and assume that $\mathcal{O}_{K}$ is Richardson in the sense of Definition 5.26 below. Write $Q \in K \backslash \mathfrak{B}$ for the orbit attached to $\mathfrak{s}$ as in Example 3.5. Then, in the notation of $\S 2.1, X(Q)=A_{\mathfrak{s}}$, and $\mathcal{O}_{K}$ is dense in $\mu\left(T_{Q}^{*} \mathfrak{B}\right)$. Hence, it follows that $M_{Q}:=X(Q)$ satisfies conditions (i) and (ii) of Hypothesis $(\star)$. This is the main trick we will use in verifying Hypothesis $(\star)$ in $\S 5$ : we start with a derived functor module where the verification of the conditions of the hypothesis is easy, and then use that information to define other (not necessarily derived functor) modules $M_{Q_{i}}$ to fulfill the hypothesis.

\section{Connection with highest weight modules}

Fix a Borel subgroup $B$ in $G$ with Lie algebra $\mathfrak{b}=\mathfrak{h} \oplus \mathfrak{n}$, and write $W$ for the Weyl group of $\mathfrak{h}$ in $\mathfrak{g}$. Let $\rho$ denote the half-sum of the positive roots of $\mathfrak{h}$ in $\mathfrak{n}$. Consider the category of finite-length $(\mathfrak{g}, B)$ modules. The irreducible objects with trivial infinitesimal character are parametrized by $W$. If $w_{\circ}$ denotes the long element in $W$, we arrange the parametrization so that $L(w)$ is the unique irreducible quotient of the Verma module $M(w):=\operatorname{ind}_{\mathfrak{b}}^{\mathfrak{g}}\left(\mathbb{C}_{w w_{\circ} \rho-\rho}\right)$.

We may easily transcribe the constructions of $\S 3$ from the category of $(\mathfrak{g}, K)$ modules to that of $(\mathfrak{g}, B)$ modules: one need only replace $K$ with $B$ in the discussion. In this case we write

$$
\operatorname{CV}(L)=\bigcup_{w \in \operatorname{cv}(L)} \overline{T_{w}^{*} \mathfrak{B}}, \quad \mathcal{C V}(L)=\sum_{w \in \operatorname{cv}(L)} m_{w} \overline{T_{w}^{*} \mathfrak{B}}
$$

Here $\operatorname{cv}(L)$ is a subset of $B$ orbits on $\mathfrak{B}$; by the Bruhat decomposition, we identify $B \backslash \mathfrak{B}$ with $W$, and write $T_{w}^{*} \mathfrak{B}$ for the conormal bundle to the orbit parametrized by $w$. Analogously, we write

$$
\operatorname{AV}(L)=\bigcup_{\mathbb{D} \in \operatorname{av}(L)} \overline{\mathbb{O}}, \quad \mathcal{A} \mathcal{V}(L)=\sum_{\mathbb{D} \in \operatorname{av}(L)} m_{\mathbb{O}} \overline{\mathbb{O}} .
$$




\section{LEADING-TERM CYCLES OF HARISH-CHANDRA MODULES}

Let $\overline{\mathcal{O}}$ denote the associated variety of $\operatorname{Ann}(L)(\S 2.2)$. Just as av $(X)$ for an irreducible $(\mathfrak{g}, K)$ module consisted of $K$ invariant Lagrangians of $\mathcal{O} \cap(\mathfrak{g} / \mathfrak{k})^{*}$, so av $(L)$ consists of $B$ invariant Lagrangians in $\mathcal{O} \cap(\mathfrak{g} / \mathfrak{b})^{*}$.

The following theorem is due to Joseph [Jos84]. His proof is algebraic in nature. A very simple geometric proof using Theorem 3.13 is indicated below.

Theorem 4.1. Consider the simple $(\mathfrak{g}, B)$ module $L(w)$ with trivial infinitesimal character. Then

$$
\operatorname{AV}(L(w))=\overline{\mathbb{O}_{1}} \cup \cdots \cup \overline{\mathbb{O}_{k}}
$$

if and only if there exist nonzero constants $c_{i}$ such that

$$
q_{\mathrm{Ann}\left(L\left(w^{-1}\right)\right)}=\sum_{i} c_{i} p_{\mathbb{O}_{i}},
$$

with the notation as in $\S \S 2.2$ and 2.6.

Sketch of the proof. Let $X(w)$ denote the irreducible Harish-Chandra module for the complex group $G$ parameterized by $w$; this is a $(\mathfrak{g} \oplus \mathfrak{g}, \Delta(G))$ module where $\Delta(G)$ denotes the diagonal copy of $G$ in $G \times G$. It is an easy consequence of the geometric equivalence of categories between Harish-Chandra modules for $G$ and highest weight modules for $\mathfrak{g}$ (e.g. Borho and Brylinski [BB85, Corollary 4.10]) that $\operatorname{AV}(X(w))=\operatorname{AV}(\operatorname{Ann}(L(w)))=: \overline{\mathcal{O}}$. (Here we are being a little sloppy: $\operatorname{AV}(X(w))$ is the closure of a nilpotent orbit of $\Delta(G)$ on $(\mathfrak{g} \oplus \mathfrak{g} / \Delta(\mathfrak{g}))^{*}$ where $\Delta(\mathfrak{g})$ denotes the diagonal copy of $\mathfrak{g}$ in $\mathfrak{g} \oplus \mathfrak{g}$, but we simply identify $\mathcal{O}$ with a nilpotent orbit of $G$ on $\mathcal{N}\left(\mathfrak{g}^{*}\right)$.) Moreover, it is easy to check that

$$
\operatorname{Ann}_{\mathrm{U}(\mathfrak{g} \oplus \mathfrak{g})}(X(w))=I\left(w^{-1}\right) \otimes \mathrm{U}(\mathfrak{g})+\mathrm{U}(\mathfrak{g}) \otimes I(w)
$$

from which one concludes that the Goldie rank polynomial of $\operatorname{Ann}(X(w))$ factors as

$$
q_{\mathrm{Ann}(X(w))}=q_{I\left(w^{-1}\right)} q_{I(w)} .
$$

Let $T_{x}^{*}(\mathfrak{B} \times \mathfrak{B})$ denote the conormal bundle to $Q(x)$, the $\Delta(G)$ orbit on $\mathfrak{B} \times \mathfrak{B}$ parameterized by $x$. (Informally $Q(x)$ consists of flags in relative position $x$.) Suppose $x \in W$ such that $\mu\left(T_{x}^{*}(\mathfrak{B} \times \mathfrak{B})\right)=\overline{\mathcal{O}}$, and fix $\xi \in \mathcal{O}$. Proposition 2.7 attaches an orbit $\Delta\left(A_{G}(\xi)\right) \cdot\left(C_{l}, C_{r}\right) \in$ $\Delta\left(A_{G}(\xi)\right) \backslash \operatorname{Irr}\left(\mu^{-1}(\xi) \times \mu^{-1}(\xi)\right)$ to $Q(x)$; here $\Delta\left(A_{G}(\xi)\right)$ denotes the diagonal copy of $\Delta\left(A_{G}(\xi)\right)$ in $A_{G}(\xi) \times A_{G}(\xi)$. Write $Z_{l}(x)=A_{G}(\xi) \cdot C_{l} \in A_{G}(\xi) \backslash \operatorname{Irr}\left(\mu^{-1}(\xi)\right)$ and similarly for $Z_{r}(x)$. From the geometric equivalence of categories, one deduces that $\mathbb{O} \in \operatorname{av}(L(w))$ if and only if there exists $Q(x) \in \operatorname{cv}(X(w) ; \mathcal{O})$ such that $Z_{l}(x)$ corresponds to $\mathbb{O}$ in the bijection of Proposition 2.3; after some unraveling, this follows, for instance, from [BB85, Theorem 4.8(a)]. According to Theorem 3.13 and the factorization of $q_{\operatorname{Ann}(X(w))}$ mentioned above, we conclude that $Q(x) \in \operatorname{cv}(X(w) ; \mathcal{O})$ if and only if the expression of $q_{I\left(w^{-1}\right)}$ in the basis $\left\{p_{Z} \mid Z \in A_{G}(\xi) \backslash \operatorname{Irr}\left(\mu^{-1}(\xi)\right)\right\}$ contains $p_{Z_{l}(x)}$ with nonzero coefficient. The last two sentences then give the conclusion of the theorem.

Using Theorem 3.13, we can thus transfer information between the Harish-Chandra category and the highest weight category.

Corollary 4.2. Fix a special nilpotent orbit $\mathcal{O}$, a real form $G_{\mathbb{R}}$ for $G$, and $\mathcal{O}_{K} \in K \backslash\left(\mathcal{O} \cap(\mathfrak{g} / \mathfrak{k})^{*}\right)$. Suppose that:

(i) $X$ is a Harish-Chandra module for $G_{\mathbb{R}}$ with trivial infinitesimal character such that $\mathcal{O}_{K} \in$ $\operatorname{av}(X) ;$ and

(ii) $L(w)$ is a simple highest weight module for $\mathfrak{g}$ with trivial infinitesimal character such that $\operatorname{Ann}\left(L\left(w^{-1}\right)\right)=\operatorname{Ann}(X)$.

Fix $Q \in \mu^{-1}\left(\mathcal{O}_{K}\right)$ (with the notation as in $\S 2.5$ ), and let $\mathbb{O}(Q)$ denote the orbital variety corresponding to $A_{G}(\xi) \cdot\left(\mu^{-1}(\xi) \cap T_{Q}^{*} \mathfrak{B}\right)$ (Propositions 2.3 and 2.7). Then

$$
Q \in \operatorname{cv}\left(X ; \mathcal{O}_{K}\right) \Longrightarrow \mathbb{O}(Q) \in \operatorname{av}(L) .
$$




\section{P. E. TRAPA}

If we further assume that the $A_{K}(\xi)$ and $A_{G}(\xi)$ orbits on $\operatorname{Irr}\left(\mu^{-1}(\xi)\right)$ coincide (so the assignment $Q \mapsto \mathbb{O}(Q)$ is bijective by Propositions 2.3 and 2.7), then

$$
Q \in \operatorname{cv}\left(X ; \mathcal{O}_{K}\right) \Longleftrightarrow \mathbb{O}(Q) \in \operatorname{av}(L) .
$$

Proof. According to Theorem 3.13, $Q \in \mathrm{cv}\left(X, \mathcal{O}_{K}\right)$ implies that $p_{Q}$ appears with nonzero coefficient in the expression of $q_{\operatorname{Ann}(X)}$ in terms of fiber polynomials. Since we have assumed that $\operatorname{Ann}(X)=$ $\operatorname{Ann}\left(L\left(w^{-1}\right)\right)$, Theorem 4.1 implies that the condition of the previous sentence implies $\mathbb{O}(Q) \in$ av $(L)$. If $A_{K}(\xi) \backslash \operatorname{Irr}\left(\mu^{-1}(\xi)\right)=A_{G}(\xi) \backslash \operatorname{Irr}\left(\mu^{-1}(\xi)\right)$, then Theorem 2.1(ii) implies $p_{Q} \neq p_{Q^{\prime}}$ for $Q \neq Q^{\prime}$ in $\mu^{-1}\left(\mathcal{O}_{K}\right)$. This gives the final conclusion of the theorem.

Note that Corollary 4.2 quantifies how difficult it is to compute leading-term cycles of a HarishChandra module $X$. Roughly speaking, one must compute the annihilator and associated variety of $X$ and then the associated variety of a single simple highest weight module.

Remark 4.3. It seems likely that the statement of Corollary 4.2 can be deduced from a general functorial relationship between Harish-Chandra modules and highest weight modules. We would like to return to this elsewhere.

\section{Hypothesis $(\star)$}

The purpose of this section is to place Hypothesis $(\star)$ (given in $\S 3.3$ ) in a broader context, as well as establish it in all classical cases.

So fix notation as in Hypothesis $(\star)$. Fix $G_{\mathbb{R}}$ such that $\mathcal{O} \cap(\mathfrak{g} / \mathfrak{k})^{*}$ is nonempty and fix $\mathcal{O}_{K}$ and $Q \in \mu^{-1}\left(\mathcal{O}_{K}\right)$. Then it is essentially obvious that we may find a virtual Harish-Chandra module $M_{Q}$ satisfying the conditions of Hypothesis $(\star)$ for $\mathcal{O}_{K}$. This is easy to arrange since the characteristic cycle construction is additive: we start with any $M_{Q}$ supported on $Q$ and subtract off appropriate Harish-Chandra modules. The techniques of $\S 3$ then relate an algebraic basis of multiplicity polynomials of the virtual Harish-Chandra modules $M_{Q}$ to the geometric basis arising in the Springer fiber. The change of basis matrix is upper triangular because of Proposition 3.2. As explained in $\S 2$, if $M_{Q}$ is irreducible (that is, if Hypothesis ( $\star$ ) holds), then the multiplicity polynomial is a Goldie rank polynomial, and hence we obtain Theorem 1.2. It seems very likely that the appropriate virtual representations may be chosen so that their multiplicity polynomials are, in fact, proportional to Goldie rank polynomials. If this is the case, Theorem 1.2 would hold without Hypothesis $(\star)$. However, we have been unable to see that the virtual Harish-Chandra modules may be chosen so that their multiplicity polynomials are in fact of the form $p_{I}$. Thus, we are left with proving Hypothesis $(\star)$.

We begin with a discussion of Type A. Given [Mel93], this is less interesting from the point of view of Tanisaki's conjecture since it has already been established in [Mel93] that the matrix in Theorem 1.2 is, in fact, diagonal. Nonetheless, the Type A case is worth understanding. For instance, using the real group $\mathrm{U}(p, q)$ one may recover the main results of [Mel93]. The case of $\mathrm{U}(p, q)$ will also be important in the proof of Theorem 5.32. The following is taken from [Tra99, Theorem 5.6]; as explained there, for $\operatorname{GL}(n, \mathbb{C})$ the result follows by combining the work of Steinberg [Ste88] and Joseph [Jos77].

Theorem 5.1. Suppose $G_{\mathbb{R}}=\operatorname{GL}(n, \mathbb{C}), \operatorname{GL}(n, \mathbb{H})$ or $\mathrm{U}(p, q)$. (In these cases all A-group orbits on irreducible components of the Springer fiber are singletons.) If $X$ is an irreducible Harish-Chandra module for $G_{\mathbb{R}}$ with trivial infinitesimal character, then

$$
\mu\left(\overline{T_{\text {supp }_{\circ}(X)^{\mathfrak{B}}}^{*}}=\mathrm{AV}(X) .\right.
$$




\section{LEADING-TERM CYCLES OF HARISH-CHANDRA MODULES}

Consequently, if $\mathcal{O}$ is any orbit such that $\mathcal{O} \cap(\mathfrak{g} / \mathfrak{k})^{*}$ is nonempty, and $\mathcal{O}_{K} \in K \backslash\left(\mathcal{O} \cap(\mathfrak{g} / \mathfrak{k})^{*}\right)$, Hypothesis ( $\star$ ) may be verified for $\mathcal{O}_{K}$ by taking, for each $Q \in \mu^{-1}\left(\mathcal{O}_{K}\right), M_{Q}$ to be any (in fact, the unique) irreducible Harish-Chandra module with trivial infinitesimal character supported on $\bar{Q}$.

By contrast [Tra99, Remark 8.9] essentially provides an orbit $Q$ for $\operatorname{GL}(n, \mathbb{R})$ for which there is no Harish-Chandra module $M_{Q}$ satisfying the requirements (i) and (ii) of the hypothesis. This indicates the subtle nature of matters.

For the groups $\operatorname{Sp}(p, q)$ and $\mathrm{SO}^{*}(2 n)$, the situation is very similar to that of Theorem 5.1.

Theorem 5.2. Suppose $G_{\mathbb{R}}=\operatorname{Sp}(p, q)$ or $\mathrm{SO}^{*}(2 n)$. (For these groups, the orbits of $A_{K}(\xi)$ on $\operatorname{Irr}\left(\mu^{-1}(\xi)\right)$ are singletons, while those of $A_{G}(\xi)$ are typically larger.) Suppose $X$ is an irreducible Harish-Chandra module with trivial infinitesimal character. Then

$$
\mu\left(\overline{\left.T_{\text {supp }_{\circ}(X)^{\mathfrak{B}}}\right)}=\operatorname{AV}(X) .\right.
$$

Consequently, if $\mathcal{O}$ is a complex nilpotent orbit for $\mathfrak{g}$ such that $\mathcal{O} \cap(\mathfrak{g} / \mathfrak{k})^{*}$ is nonempty, $\mathcal{O}_{K} \in$ $K \backslash\left(\mathcal{O} \cap(\mathfrak{g} / \mathfrak{k})^{*}\right)$, and $\mu^{-1}\left(\mathcal{O}_{K}\right)^{\prime}$ is any subset of $\mu^{-1}\left(\mathcal{O}_{K}\right)$ of minimal type, then Hypothesis ( $\star$ ) may be verified for $\mathcal{O}_{K}$ by taking, for each $Q \in \mu^{-1}\left(\mathcal{O}_{K}\right)^{\prime}, M_{Q}$ to be any (in fact, the unique) irreducible Harish-Chandra module supported on $\bar{Q}$.

Proof. The proof is a rather elaborate counting argument using [McG98b, Theorems 6 and 10]. Given a Harish-Chandra module $X$ for $G_{\mathbb{R}}$, let $\mathcal{C}(X)$ denote the cell of Harish-Chandra modules containing $X$ (e.g. [McG98b, $\S 1]$ ). Write $G$ for the complexification of $G_{\mathbb{R}}$. For the groups in question, all Cartan subgroups are connected. So the only $K$-equivariant local system supported on a fixed orbit $Q \in K \backslash \mathfrak{B}$ is the trivial one. As in the notation of $\S 2.1$, we set $X(Q)=X(Q, \mathbb{1})$.

The first ingredient we need is the inclusion

$$
\overline{G \cdot \mu(Q)} \subset \operatorname{AV}(\operatorname{Ann}(X(Q))) ;
$$

here $\mu(Q)$ is defined as in (2.4). The conclusion of (5.4) follows from (3.3), Proposition 3.10, and the well-known fact (e.g. [Vog91, Theorem 8.4]) that

$$
G \cdot \operatorname{AV}(X)=\operatorname{AV}(\operatorname{Ann}(X)) .
$$

Now fix $Q \in K \backslash \mathfrak{B}$, set $\mathcal{O}_{K}=\mu(Q)$ (with the notation as in (2.4)), and fix $\xi \in \mathcal{O}_{K}$. By imitating the explicit centralizer calculations given in [CM93, $\S 6.1$, one quickly concludes that the image of $A_{K}(\xi)$ in $A_{G}(\xi)$ is trivial. Hence, by Proposition 2.7,

$$
\# \mu^{-1}\left(\mathcal{O}_{K}\right)=\# \operatorname{Irr}\left(\mu^{-1}(\xi)\right)
$$

with the notation as in $\S 2.5$. On the other hand, set $\mathcal{C}(Q)=\mathcal{C}(X(Q))$, the cell containing $X(Q)$. In the terminology of [McG98b], $\mathcal{C}(Q)$ is Springer in the sense that the cell affords the same Weyl group representations as the representation on the top homology of the entire Springer fiber; see [McG98b, Theorems 6 and 10]. This implies that

$$
\# \mathcal{C}(Q)=\# \operatorname{Irr}\left(\mu^{-1}(\xi)\right),
$$

and so

$$
\# \mathcal{C}(Q)=\# \mu^{-1}\left(\mathcal{O}_{K}\right)
$$

Let $N$ denote this number. Set $\mathcal{O}=G \cdot \mathcal{O}_{K}$. For the groups in question, it follows from the partition classification (see Remark 5.25 below) that $\mathcal{O}$ is special. Enumerate the orbits of $K$ on $\mathcal{O} \cap(\mathfrak{g} / \mathfrak{k})^{*}$ as $\mathcal{O}_{K}^{1}, \ldots, \mathcal{O}_{K}^{k}$. Since

$$
\mu^{-1}(\mathcal{O}):=\{Q \mid G \cdot \mu(Q)=\mathcal{O}\}=\mu^{-1}\left(\mathcal{O}_{K}^{1}\right) \cup \cdots \cup \mu^{-1}\left(\mathcal{O}_{K}^{k}\right),
$$




\section{P. E. TRAPA}

Equation (5.6) implies

$$
\#\{Q \mid G \cdot \mu(Q)=\mathcal{O}\}=k N .
$$

One the other hand, consider the set of Harish-Chandra modules

$$
\{X \mid \operatorname{AV}(\operatorname{Ann}(X))=\overline{\mathcal{O}}\} .
$$

From general principles, this set is a union of cells, say $\mathcal{C}_{1}, \ldots, \mathcal{C}_{l}$, each of whose corresponding representation contains the special representation $\operatorname{Sp}(\mathcal{O})$; moreover, each cell whose corresponding representation contains $\operatorname{Sp}(O)$ is among the $\mathcal{C}_{i}$. McGovern proves that each cell contains $\operatorname{Sp}(\mathcal{O})$ exactly once [McG98b, Theorem 1]. Meanwhile, it is not difficult to compute the multiplicity of $\operatorname{Sp}(\mathcal{O})$ in the full coherent continuation representation on the Grothendieck group of Harish-Chandra modules with trivial infinitesimal character. This is done in [McG98b, Theorems 6 and 10], and the multiplicity turns out to be exactly $k=\# K \backslash\left(\mathcal{O} \cap(\mathfrak{g} / \mathfrak{k})^{*}\right)$. Combining the last few sentences implies that $k=l$. Consequently, $\{X \mid \operatorname{AV}(\operatorname{Ann}(X))=\overline{\mathcal{O}}\}$ is a union of $k$ cells, each of size $N$ (by (5.8)), and we conclude from (5.10) that

$$
\#\{Q \mid G \cdot \mu(Q)=\mathcal{O}\}=\#\{X \mid \operatorname{AV}(\operatorname{Ann}(X))=\overline{\mathcal{O}}\}=k N .
$$

As we have remarked above, all Cartan subgroups for the groups in question are connected, so the map $Q \mapsto X(Q)$ is a bijection, and we may recast (5.11) as

$$
\#\{Q \mid G \cdot \mu(Q)=\mathcal{O}\}=\#\{Q \mid \operatorname{AV}(\operatorname{Ann}(X(Q)))=\overline{\mathcal{O}}\}=k N .
$$

This equality will be important below.

We now seek to establish (for the groups in question) that equality actually holds in (5.4); that is, we now show

$$
\overline{G \cdot \mu(Q)}=\operatorname{AV}(\operatorname{Ann}(X(Q))) .
$$

Suppose (5.13) fails. Let $Q$ denote an orbit for which

$$
\overline{G \cdot \mu(Q)} \subsetneq \operatorname{AV}(\operatorname{Ann}(X(Q))) .
$$

Assume $\mu(Q)$ has minimal dimension among all orbits for which the failure (5.14) holds. By assumption,

$$
S_{1}:=\left\{Q^{\prime} \mid G \cdot \mu\left(Q^{\prime}\right)=G \cdot \mu(Q)\right\}
$$

is not contained in

$$
S_{2}:=\left\{Q^{\prime} \mid \operatorname{AV}\left(\operatorname{Ann}\left(X\left(Q^{\prime}\right)\right)\right)=\overline{G \cdot \mu(Q)}\right\}
$$

since $Q$ is contained in the former set but not the latter. However, we have seen (in (5.12)) that $S_{1}$ and $S_{2}$ have the same size. Thus, there must exist an element $Q^{\prime \prime} \in S_{2}$ such that $Q^{\prime \prime} \notin S_{1}$. Since $Q^{\prime \prime}$ is in $S_{2}$,

$$
\overline{G \cdot \mu(Q)}=\operatorname{AV}\left(\operatorname{Ann}\left(X\left(Q^{\prime \prime}\right)\right)\right) .
$$

Combined with (5.4), we conclude that

$$
G \cdot \mu\left(Q^{\prime \prime}\right) \subset \operatorname{AV}\left(\operatorname{Ann}\left(X\left(Q^{\prime \prime}\right)\right)\right)=\overline{G \cdot \mu(Q)} .
$$

Since $Q^{\prime \prime}$ does not belong to $S_{1}$, we conclude that

$$
G \cdot \mu\left(Q^{\prime \prime}\right) \text { is properly contained in } \operatorname{AV}\left(\operatorname{Ann}\left(X\left(Q^{\prime \prime}\right)\right)\right)=\overline{G \cdot \mu(Q)} \text {. }
$$

However, now (5.15) and (5.16) contradict the assumption that $\mu(Q)$ had minimal dimension among all orbit for which the failure (5.14) holds. This contradiction thus establishes (5.13).

We now turn to proving the first assertion of the theorem. Fix $\mathcal{O}$ so that $K \backslash\left(\mathcal{O} \cap(\mathfrak{g} / \mathfrak{k})^{*}\right)=$ $\left\{\mathcal{O}_{K}^{1}, \ldots, \mathcal{O}_{K}^{k}\right\}$ is nonempty. Define $\mu^{-1}(\mathcal{O})$ as in (5.9). Now (5.5) and (5.13) imply that, for any $Q$,

$$
\mu(Q) \text { is dense in an irreducible component of } \operatorname{AV}(X(Q)) \text {, }
$$




\section{LEADING-TERM CYCLES OF HARISH-CHANDRA MODUlES}

in the notation of $\S 3.2$. Now choose $Q_{1}$ of minimal dimension in $\mu^{-1}(\mathcal{O})$. We claim that indeed

$$
\operatorname{AV}\left(X\left(Q_{1}\right)\right)=\overline{\mu\left(Q_{1}\right)} .
$$

Suppose $Q_{1}^{\prime}$ is an element of $\operatorname{cv}\left(X\left(Q_{1}\right)\right)$ not equal to $Q_{1}$. Then, by Proposition 3.2, $Q_{1}^{\prime} \subsetneq \overline{Q_{1}}$. Since $Q_{1}$ has minimal dimension in $\mu^{-1}(\mathcal{O}), Q_{1}^{\prime} \notin \mu^{-1}(\mathcal{O})$. Now Proposition 3.10 implies (5.18).

Let $\mathcal{C}_{1}$ denote the cell containing $X\left(Q_{1}\right)$. Since the associated varieties of two elements in the same cell are the same (see, e.g., the proof of [BB82, Lemma 4.1]), it follows that $\operatorname{AV}(X)=\overline{\mu\left(Q_{1}\right)}$ for all $X \in \mathcal{C}_{1}$, and indeed

$$
\mathcal{C}_{1} \subset\left\{X(Q) \mid \mu(Q)=\mu\left(Q_{1}\right)\right\} .
$$

However, in fact, (5.8) implies that this inclusion must be an equality,

$$
\mathcal{C}_{1}=\left\{X(Q) \mid \mu(Q)=\mu\left(Q_{1}\right)\right\} .
$$

Let $\mathcal{O}_{1}$ be the dense orbit in $\mu\left(Q_{1}\right)$. Combining (5.18) and (5.19) with the fact that associated varieties are constant on cells, we obtain

$$
\mathcal{C}_{1}=\left\{X \mid \operatorname{AV}(X)=\overline{\mathcal{O}_{1}}\right\}
$$

or, after unwinding the notation,

$$
\text { for all } X \in \mathcal{C}_{1}, \quad \operatorname{AV}(X)=\overline{\mu\left(T_{\text {supp } \left._{\circ}(X)^{\mathfrak{B}}\right)},\right.}
$$

the conclusion of the theorem for the cell $\mathcal{C}_{1}$.

Next choose $Q_{2}$ of minimal dimension in

$$
\mu^{-1}(\mathcal{O}) \backslash \mu^{-1}\left(\mathcal{O}_{1}\right)
$$

Let $X_{2}=X\left(Q_{2}\right)$ and write $\mathcal{C}_{2}$ for the cell containing it. Arguing as in (5.17), we conclude that $\overline{\mu\left(Q_{2}\right)}$ is an irreducible component of $\operatorname{AV}\left(X_{2}\right)$ and arguing as in (5.18), we conclude that the only other possible irreducible component of $\operatorname{AV}\left(X_{2}\right)$ is $\overline{\mu\left(Q_{1}\right)}$. Hence,

$$
\mathcal{C}_{2} \subset\left\{X(Q) \mid \mu(Q) \in\left\{\mu\left(Q_{1}\right), \mu\left(Q_{2}\right)\right\}\right\},
$$

and (5.19) then implies that

$$
\mathcal{C}_{2} \subset \mathcal{C}_{1} \cup\left\{X(Q) \mid \mu(Q)=\mu\left(Q_{2}\right)\right\} .
$$

Since cells are disjoint, we conclude that

$$
\mathcal{C}_{2}=\left\{X(Q) \mid \mu(Q)=\mu\left(Q_{2}\right)\right\} .
$$

Thus, we may argue as in (5.20) and (5.21) to conclude that

$$
\mathcal{C}_{2}=\left\{X \mid \operatorname{AV}(X)=\overline{\mathcal{O}_{2}}\right\}
$$

or, after unwinding the notation,

$$
\text { for all } X \in \mathcal{C}_{2}, \quad \operatorname{AV}(X)=\overline{\mu\left(T_{\text {supp }_{\circ}(X)^{\mathfrak{B})}},\right.}
$$

the conclusion of the theorem for the cell $\mathcal{C}_{1}$.

Clearly we may continue in the this way and establish the analogs of (5.21) and (5.23) for any cell. Thus, the first assertion of the theorem follows. The final assertion follows easily from the former.

Although it is not relevant to Hypothesis $(\star)$, we isolate the following interesting consequence of the above proof. 


\section{P. E. TRAPA}

Corollary 5.24. Let $G_{\mathbb{R}}=\operatorname{Sp}(p, q)$ or $\mathrm{SO}^{*}(2 n)$ and retain the notation introduced at the beginning of the proof of Theorem 5.2. Fix $\mathcal{O}_{K} \in K \backslash \mathcal{N}_{\mathfrak{p}}^{*}$. Then the set of irreducible Harish-Chandra modules $X$ with trivial infinitesimal character such that $\operatorname{AV}(X)=\overline{\mathcal{O}_{K}}$ is a cell of Harish-Chandra modules. Moreover, all cells arise in this way. In particular, if $X$ is an irreducible Harish-Chandra module for $G_{\mathbb{R}}$ with integral infinitesimal character, the associated variety of $X$ is irreducible.

Proof. The first two assertions follow from the general analogs of (5.20) and (5.22) established by the preceding proof. The final assertion is obvious for trivial infinitesimal character, and the general integral case follows from the final assertion of Proposition 3.10.

Remark 5.25. We make the hypothesis that $\mathcal{O} \cap(\mathfrak{g} / \mathfrak{k})^{*}$ be nonempty in Theorem 5.2 more explicit. As remarked in the introduction, nilpotent orbits for $G=\operatorname{Sp}(2 n, \mathbb{C})$ are parametrized by partitions of $n$ in which odd parts occur with even multiplicities. Fix such a partition $\lambda$, and write $\mathcal{O}$ for the corresponding nilpotent orbit. Then there exists a real form $\operatorname{Sp}(p, q)$ of $G$ such that $\mathcal{O} \cap(\mathfrak{g} / \mathfrak{k})^{*}$ is nonempty if and only if every part of $\lambda$ occurs with even multiplicity. (In this case $\mathcal{O}$ is automatically special since an orbit is special if it corresponds to a partition in which the number of even parts between consecutive odd parts or greater than the largest odd part is even.) Meanwhile nilpotent orbits for $\mathrm{O}(2 n, \mathbb{C})$ are parametrized by partitions of $2 n$ in which even parts have even multiplicity. Fix such a partition $\lambda$ and let $\mathcal{O}$ for the corresponding orbit. Fix $\mathfrak{p}$ arising from the real form $\mathrm{SO}^{*}(2 n)$. Then $\mathcal{O} \cap(\mathfrak{g} / \mathfrak{k})^{*}$ if and only if each part of $\lambda$ occurs with even multiplicity. (Again this implies that $\mathcal{O}$ is special.)

In view of Remark 5.25, Theorem 5.2 falls short of verifying Hypothesis $(\star)$ for all orbits $\mathcal{O}$ in Types $\mathrm{C}$ and D. We now sketch a complete treatment of Types B, C, and D using the groups $\mathrm{SO}(p, q)$ and $\mathrm{Sp}(2 n, \mathbb{R})$. (The Type $\mathrm{D}$ case is due to unpublished work of W. McGovern.) As alluded to in Example 3.21, we need the following definition.

Definition 5.26. Fix $G_{\mathbb{R}}$ and the notation of $\S 2.1$. An orbit $\mathcal{O}_{K} \in K \backslash\left(\mathcal{O} \cap(\mathfrak{g} / \mathfrak{k})^{*}\right)$ is called Richardson if there exists a $\theta$-stable parabolic $\mathfrak{s}=\mathfrak{l} \oplus \mathfrak{u}$ such that $\mathcal{O}_{K}$ is dense in $K \cdot \mathfrak{u}$. (If $G_{\mathbb{R}}$ is a complex group, this latter condition reduces to the usual notion of complex Richardson orbits defined, for example, in [CM93, §7.1].) As explained in the introduction of [Tra05b], if $\mathcal{O}_{K}$ is Richardson, then $G \cdot \mathcal{O}_{K}$ is automatically special.

We need to make this definition explicit for applications. We begin with $G_{\mathbb{R}}=\operatorname{Sp}(2 n, \mathbb{R})$. Fix $\mathcal{O}$ parametrized by a partition $\lambda$ as in Remark 5.25. Then, according to [CM93, Theorem 9.3.5], the elements of $K \backslash\left(\mathcal{O} \cap(\mathfrak{g} / \mathfrak{k})^{*}\right)$ are parametrized by diagrams obtained by filling the boxes of $\lambda$ (viewed as a Young diagram) with plus and minus signs so as to alternate across rows and so that the number of plus signs which begin rows of each odd length $2 k+1$ coincides with the number of minus signs that begin rows of length $2 k+1$, modulo the equivalence of interchanging rows of equal length. Hence, $\mathcal{O} \cap(\mathfrak{g} / \mathfrak{k})^{*}$ is always nonempty.

Proposition 5.27 (see [Tra05b, Corollary 4.2]). Let $G_{\mathbb{R}}=\operatorname{Sp}(2 n, \mathbb{R})$. Fix a complex special orbit $\mathcal{O}$. Consider $\mathcal{O}^{K} \in K \backslash\left(\mathcal{O} \cap(\mathfrak{g} / \mathfrak{k})^{*}\right)$ parametrized, as described above, by a signed diagram $\lambda_{ \pm}$. Fix a chunk of even rows between consecutive odd rows or greater than the largest odd row, and enumerate the distinct parts of this chunk as $2 k_{1}, \ldots, 2 k_{r}$. Then the orbit $\mathcal{O}^{K}$ is Richardson (Definition 5.26) if and only if the following two conditions are satisfied for each such maximal chunk of even rows:

(i) each row of length $2 k_{i}$ begins with the same sign $\varepsilon_{i}$; and

(ii) there is a fixed sign $\varepsilon$ (depending on the chunk of even rows being considered) such that

$$
\varepsilon_{i}=\varepsilon(-1)^{k_{i}} .
$$

Note that, for any special orbit $\mathcal{O}$, there exists an element of $K \backslash\left(\mathcal{O} \cap(\mathfrak{g} / \mathfrak{k})^{*}\right)$ which is Richardson. 


\section{LEADING-TERM CYCLES OF HARISH-CHANDRA MODULES}

It is perhaps useful to give a slightly less combinatorial interpretation of the explicit Richardson condition appearing in the proposition. Fix an arbitrary orbit $\mathcal{O}_{K}$ parametrized by $\lambda_{ \pm}$, and let $Z\left(\mathcal{O}_{K}\right)$ denote the centralizer in $K$ of a point of $\mathcal{O}_{K}$. (So $Z\left(\mathcal{O}_{K}\right)$ is well defined up to isomorphism.) The group $Z\left(\mathcal{O}_{K}\right)$ factors as a direct product indexed by the distinct parts of the underlying partition $\lambda$. Suppose $d$ is such a part that occurs with multiplicity $m$. If $m=2 l+1$ is odd, then the part $d$ contributes a factor $O(l, l+1)$ to $Z\left(\mathcal{O}_{K}\right)$. If $m=2 k$ is odd, write $k_{+}$(respectively $k_{-}$) for the number of plus (respectively minus) signs at the beginning of the rows of length $2 k$ in $\lambda_{ \pm}$. Then the part $m$ contributes a factor $\operatorname{Sp}\left(k_{+}, k_{-}\right)$to the centralizer $Z\left(\mathcal{O}_{K}\right)$. Hence, part (i) of the Richardson condition is that the centralizer of an element of $\mathcal{O}_{K}$ is, in an appropriate sense, as compact as possible; part (ii) is a requirement on the signatures of the compact factors of the centralizer.

We will ultimately verify Hypothesis $(\star)$ for orbits $\mathcal{O}_{K}$ which are a special kind of Richardson orbit. The following is the definition we need.

Definition 5.28. Let $G_{\mathbb{R}}=\operatorname{Sp}(2 n, \mathbb{R})$. Fix a complex special orbit $\mathcal{O}$. Consider $\mathcal{O}^{K} \in K \backslash(\mathcal{O} \cap$ $\left.(\mathfrak{g} / \mathfrak{k})^{*}\right)$ to be parametrized, as described above, by a signed diagram $\lambda_{ \pm}$. The orbit is called a relevant orbit if it is Richardson (Definition 5.26 and Proposition 5.27) and additionally, if $\lambda$ has only even parts, the condition of Proposition 5.27 applies to the chunk consisting of all its even parts. (This latter condition is of course a strengthening of the Richardson one.) Note that, for any special orbit $\mathcal{O}$, there exists an element of $K \backslash\left(\mathcal{O} \cap(\mathfrak{g} / \mathfrak{k})^{*}\right)$ which is relevant.

Once again, one may provide a slightly more intrinsic definition of relevant by translating it into a condition on the compact part of the centralizer of a point in $\mathcal{O}_{K}$.

We need the analogous definitions for the groups $G_{\mathbb{R}}=\mathrm{O}(p, q)$. (The combinatorics of the full (disconnected) orthogonal group, as usual, is slightly simpler, although we could easily work with $\mathrm{SO}(p, q)$ or its identity component instead.) This time complex nilpotent orbits for $\mathfrak{g}$ are parametrized by partitions $\lambda$ in which every even part occurs an even number of times. If $p+q$ is odd (respectively even), an orbit is special if its corresponding partition has an even number of odd parts between consecutive even parts and an odd (respectively even) number of odd parts greater than the largest even part.

Fix $\mathcal{O}$ corresponding to $\lambda$. Then, according to [CM93, Theorem 9.3.4], the elements of $K \backslash(\mathcal{O} \cap$ $\left.(\mathfrak{g} / \mathfrak{k})^{*}\right)$ are parametrized by diagrams obtained by filling the boxes of $\lambda$ (viewed as a Young diagram) with $p$ plus and $q$ minus signs so as to alternate across rows and so that the number of plus signs which begin rows of each even length $2 k$ coincides with the number of minus signs that begin rows of length $2 k$, modulo the equivalence of interchanging rows of equal length. Hence, for the split groups $\mathrm{O}(n, n+1)$ and $\mathrm{O}(n, n), \mathcal{O} \cap(\mathfrak{g} / \mathfrak{k})^{*}$ is always nonempty.

Proposition 5.29 (See [Tra05b, Corollary 7.2]). Let $G_{\mathbb{R}}=\mathrm{O}(p, q)$. Fix a complex special orbit $\mathcal{O}$. Consider $\mathcal{O}^{K} \in K \backslash\left(\mathcal{O} \cap(\mathfrak{g} / \mathfrak{k})^{*}\right)$ parametrized, as described above, by a signed diagram $\lambda_{ \pm}$. Fix a maximal chunk of odd rows between consecutive even parts, and enumerate the distinct parts of this chunk as $2 k_{1}+1, \ldots, 2 k_{r}+1$. The orbit $\mathcal{O}^{K}$ is Richardson (Definition 5.26) if and only if the following two conditions are satisfied for each maximal chunk of odd rows:

(i) each row of length $2 k_{i}+1$ begins with the same sign $\varepsilon_{i}$; and

(ii) there is a fixed sign $\varepsilon$ (depending on the chunk of odd rows being considered) such that

$$
\varepsilon_{i}=\varepsilon(-1)^{k_{i}} .
$$

Note that for the split groups $\mathrm{O}(n, n+1)$ and $\mathrm{O}(n, n)$, given any special orbit $\mathcal{O}$, there exists an element of $K \backslash\left(\mathcal{O} \cap(\mathfrak{g} / \mathfrak{k})^{*}\right)$ which is Richardson. 


\section{P. E. TRAPA}

Once again, the definition translates into a condition on the compact part of the centralizer. We omit the details.

Definition 5.30. Let $G_{\mathbb{R}}=\mathrm{O}(p, q)$. Fix a complex special orbit $\mathcal{O}$. Consider $\mathcal{O}^{K} \in K \backslash\left(\mathcal{O} \cap(\mathfrak{g} / \mathfrak{k})^{*}\right)$ parametrized, as described above, by a signed diagram $\lambda_{ \pm}$. The orbit $\mathcal{O}^{K}$ is called a relevant orbit if it is Richardson (Definition 5.26 and Proposition 5.29) and additionally, if $\lambda$ has only odd parts, the condition of Proposition 5.29 applies to the chunk consisting of all its odd parts. Note that for any special orbit $\mathcal{O}$ such that $\mathcal{O} \cap(\mathfrak{g} / \mathfrak{k})^{*}$ is nonempty (which is always the case if $G_{\mathbb{R}}$ is split), there exists an element of $K \backslash\left(\mathcal{O} \cap(\mathfrak{g} / \mathfrak{k})^{*}\right)$ which is relevant.

Now fix $G_{\mathbb{R}}^{\prime}=\operatorname{SO}(p, q)$ and a complex special orbit $\mathcal{O}^{\prime}$ for $\mathfrak{g}^{\prime}$. An orbit $\mathcal{O}_{K}^{\prime} \in K^{\prime} \backslash\left(\mathcal{O}^{\prime} \cap\left(\mathfrak{g}^{\prime} / \mathfrak{k}^{\prime}\right)^{*}\right)$ is called a relevant orbit for $\mathrm{SO}(p, q)$ if and only if $K \cdot \mathcal{O}_{K}^{\prime}$ is a relevant orbit for $\mathrm{O}(p, q)$.

The next result simplifies matters slightly for the groups $\operatorname{Sp}(2 n, \mathbb{R})$ and $\mathrm{O}(p, q)$.

Lemma 5.31. Let $G_{\mathbb{R}}=\operatorname{Sp}(2 n, \mathbb{R})$ or $\mathrm{O}(p, q)$ and fix a nilpotent element $\xi \in(\mathfrak{g} / \mathfrak{k})^{*}$. Then the natural map

$$
A_{K}(\xi) \longrightarrow A_{G}(\xi)
$$

is always surjective. In particular, the orbits of $A_{K}(\xi)$ and $A_{G}(\xi)$ on $\operatorname{Irr}\left(\mu^{-1}(\xi)\right)$ coincide.

Proof. This may be verified by a direct computation of centralizers along the lines of [CM93, ch. 6]. We omit the details.

Theorem 5.32. Let $G_{\mathbb{R}}=\operatorname{Sp}(2 n, \mathbb{R})$ or $\mathrm{SO}(p, q)$ with $p+q$ odd. Fix a complex special orbit $\mathcal{O}$ such that $\mathcal{O} \cap(\mathfrak{g} / \mathfrak{k})^{*}$ is nonempty. (This is always the case if $G_{\mathbb{R}}$ is split.) Fix a relevant orbit $\mathcal{O}_{K} \in \backslash\left(\mathcal{O} \cap(\mathfrak{g} / \mathfrak{k})^{*}\right)$ (Definitions 5.28 and 5.30); as remarked above, if $\mathcal{O} \cap(\mathfrak{g} / \mathfrak{k})^{*}$ is nonempty, then such a relevant orbit exists. For any $Q \in \mu^{-1}\left(\mathcal{O}_{K}\right)$, write (as in $\left.\S 2.1\right) X(Q)$ for the irreducible HarishChandra module attached to the trivial local system on $Q$; so, in particular, $\operatorname{supp}_{\circ}(X(Q))=Q$. Then

$$
\mu\left(\overline{T_{Q}^{*} \mathfrak{B}}\right)=\operatorname{AV}(X(Q))=\overline{\mathcal{O}_{K}}
$$

Thus, Hypothesis ( $\star$ ) may be verified by taking $M_{Q}=X(Q)$ for each $Q \in \mu^{-1}\left(\mathcal{O}_{K}\right)$.

Remark 5.33. Example 3.5.2 in [Tra05a] shows that the relevant hypothesis on $\mathcal{O}_{K}$ is necessary in general.

Remark 5.34. As the proof below shows, each module $M_{Q}$ appearing in the statement of the theorem lies in the same Harish-Chandra cell as a module of the form $A_{\mathfrak{s}}$ for a $\theta$-stable parabolic $\mathfrak{s}=\mathfrak{l} \oplus \mathfrak{u}$ where $\mathcal{O}_{K}$ is dense in $K \cdot \mathfrak{u}$.

Sketch of the proof of Theorem 5.32. Lemma 5.31 simplifies notation somewhat, for example $\mu^{-1}\left(\mathcal{O}_{K}\right)$ is the only subset of minimal type appearing in Hypothesis $(\star)$, and $\# \mu^{-1}\left(\mathcal{O}_{K}\right)$ coincides with the dimension of $\operatorname{Sp}(\mathcal{O})$ (by Proposition 2.7). We will prove the theorem for $\operatorname{Sp}(2 n, \mathbb{R}$ ). The modifications necessary for $\mathrm{SO}(p, q)$ are straightforward.

Fix $\mathcal{O}$ and choose $\mathcal{O}_{K} \in K \backslash\left(\mathcal{O} \cap(\mathfrak{g} / \mathfrak{k})^{*}\right)$ relevant. Since $\mathcal{O}_{K}$ is relevant, it is Richardson, and there exists a $\theta$-stable $\mathfrak{s}=\mathfrak{l} \oplus \mathfrak{u}$ with $\mathcal{O}_{K}$ dense in $K \cdot \mathfrak{u}$. Let $Q \in K \backslash \mathfrak{B}$ be the orbit attached to $\mathfrak{s}$ according to Example 3.5. Example 3.21 shows that we may take $M_{Q}:=X(Q)=A_{\mathfrak{s}}$ to satisfy conditions (i) and (ii) of Hypothesis $(\star)$ for the orbit $Q$.

We will define the other modules $M_{Q_{i}}$ by applying a sequence of operators of the form $T_{\alpha \beta}$ to $M_{Q}=X(Q)$. These operators are introduced in [Vog79a, §3] on the level of primitive ideals, and their definitions easily extend to Harish-Chandra modules using [Vog79b, Theorem 3.10]. See also [Gar90, Gar92, Gar93a, McG98a, McG98b, GV92] for further details. Their domains and ranges may be specified using the Borho-Jantzen-Duflo $\tau$-invariant (see [Vog79b, Definition 3.3] 


\section{LEADING-TERM CYCLES OF HARISH-CHANDRA MODULES}

and [Gar90, Gar92, Gar93a]), which is defined for Harish-Chandra modules or primitive ideals with trivial infinitesimal character. They are single-valued (respectively potentially double valued) if $\alpha$ and $\beta$ have equal (respectively unequal) length. If $Y \in T_{\alpha \beta}(X)$ and $X$ and $Y$ are Harish-Chandra modules, then $X$ and $Y$ belong to the same cell and hence, as mentioned in the proof of Theorem 5.2, $\mathrm{AV}(X)=\mathrm{AV}(Y)$. In the course of verifying Hypothesis $(\star)$ for the modules $M_{Q_{i}}$ we will also calculate the annihilators and associated varieties of these modules, using algorithms introduced in [Tra05a, §3].

Set $M_{Q}=X(Q)$, the derived functor module fixed above. Suppose $\mathbf{T}=T_{\alpha_{k}, \beta_{k}} \circ \cdots \circ T_{\alpha_{1} \beta_{1}}$ is a sequence of $T_{\alpha \beta}$ operators so that $\mathbf{T}\left(M_{Q}\right)$ is defined. Write $\mathbf{I}(Q)$ for the set of primitive ideals obtained as annihilators of elements in $\mathbf{T}\left(M_{Q}\right)$. Suppose that

$$
\begin{aligned}
& \text { for all } I \in \mathbf{I}(Q) \text {, } \\
& \quad \text { there exists } Q_{1} \in \mu^{-1}\left(\mathcal{O}_{K}\right) \text { such that } \operatorname{Ann}\left(X\left(Q_{1}\right)\right)=I \text { and } M_{Q_{1}}:=X\left(Q_{1}\right) \in \mathbf{T}\left(M_{Q}\right)
\end{aligned}
$$

with the notation as in $\S 2.1$. Then since $\operatorname{supp}_{\circ}\left(M_{Q_{1}}\right)=Q_{1}$ and since (as we remarked above) $\mathbf{T}$ preserves associated varieties (so that $\operatorname{AV}\left(M_{Q_{1}}\right)=\operatorname{AV}\left(M_{Q}\right)=\overline{\mathcal{O}_{K}}$ ), it follows that $M_{Q_{1}}$ satisfies conditions (i) and (ii) of Hypothesis $(\star)$ for the orbit $Q_{1}$. If we can make the corresponding conclusion for all well-defined sequences $\mathbf{T}$, then it follows that Hypothesis $(\star)$ holds for $\mathcal{O}_{K}$ : it follows from general principles that the collection of modules $M_{Q_{1}}$ obtained from all such sequences $\mathbf{T}$ corresponds to a submodule of the special representation $\operatorname{Sp}(\mathcal{O})$; since $\operatorname{Sp}(\mathcal{O})$ is irreducible, the submodule must be all of $\operatorname{Sp}(\mathcal{O})$; and, finally, since $\# \mu^{-1}\left(\mathcal{O}_{K}\right)$ coincides with the dimension of $\operatorname{Sp}(\mathcal{O})$ (as mentioned above), it follows that conditions (i) and (ii) in Hypothesis $(\star)$ hold for every orbit in $\mu^{-1}\left(\mathcal{O}_{K}\right)$ and hence Hypothesis $(\star)$ holds. The remaining assertions in the theorem are then obvious.

Thus, our task is to establish (5.35) for every possible sequence T. Clearly by induction it suffices to do this for a single operator $T_{\alpha \beta}$. More precisely, if $Q \in \mu^{-1}\left(\mathcal{O}_{K}\right)$ is an orbit for which $X(Q)$ satisfies condition (ii) of Hypothesis $(\star)$, write $I_{\alpha \beta}(Q)$ for the (one or two element) set of primitive ideals obtained as annihilators of elements of $T_{\alpha \beta}(X(Q))$ (when defined). Then we are to show

$$
\text { for all } I \in I_{\alpha \beta}(Q) \text {, }
$$

there exists $Q_{1} \in \mu^{-1}\left(\mathcal{O}_{K}\right)$ such that $\operatorname{Ann}\left(X\left(Q_{1}\right)\right)=I$ and $M_{Q_{1}}:=X\left(Q_{1}\right) \in T_{\alpha \beta}\left(M_{Q}\right)$,

for each $T_{\alpha \beta}$ for which $T_{\alpha \beta}\left(M_{Q}\right)$ is defined. (Here $Q$ now denotes an arbitrary element of $\mu^{-1}\left(\mathcal{O}_{K}\right)$, not the orbit corresponding to $A_{\mathfrak{s}}$.)

We need to recall the computation of moment map images of conormal bundles given in [Tra05a]. For this we need the group $G_{\mathbb{R}}^{\prime}=\mathrm{U}(n, n)$. Adopt the usual notation for $G_{\mathbb{R}}^{\prime}$ with the appropriate addition of primes; for instance, we will consider $\mathfrak{B}^{\prime}$, the flag variety for $\mathfrak{g}^{\prime} \simeq \mathfrak{g l}(2 n, \mathbb{C})$, with moment map $\mu^{\prime}$. Embed $G_{\mathbb{R}}$ in $G_{\mathbb{R}}^{\prime}$ in the obvious way. Fix a Cartan involution $\theta^{\prime}$ for $G_{\mathbb{R}}^{\prime}$ and let $\theta$ denote its restriction to $G_{\mathbb{R}}$, a Cartan involution for $G_{\mathbb{R}}$. Then $K$ is a subgroup of $K^{\prime}$. Fix a Cartan subalgebra $\mathfrak{h}^{\prime}$ for $\mathfrak{g}^{\prime}$ and let $\mathfrak{h}=\mathfrak{h}^{\prime} \cap \mathfrak{g}$; this is a Cartan subalgebra for $\mathfrak{g}$.

Since $K$ is a subgroup of $K^{\prime}$, to each orbit $Q \in K \backslash \mathfrak{B}$, we may consider the well-defined orbit

$$
Q^{\prime}:=K^{\prime} \cdot Q \in K^{\prime} \backslash \mathfrak{B}^{\prime} .
$$

The (injective) map $Q \mapsto Q^{\prime}$ is computed explicitly in [Tra05b, $\S 2.11$ ]. In addition, [Tra05a, Proposition 3.3.1] implies that

with notation as in (2.5).

$$
K^{\prime} \cdot \mu(Q)=\mu^{\prime}\left(Q^{\prime}\right)
$$

Suppose $\alpha$ is a short root for $\mathfrak{h}$ in $\mathfrak{g}$. There are two roots, say $\alpha_{1}^{\prime}$ and $\alpha_{2}^{\prime}$, for $\mathfrak{h}^{\prime}$ in $\mathfrak{g}^{\prime}$ which coincide with $\alpha$ when restricted to $\mathfrak{h}$. Meanwhile, if $\gamma$ is a long root of $\mathfrak{h}$ in $\mathfrak{g}$, there is a unique root, say $\gamma^{\prime}$, of $\mathfrak{h}^{\prime}$ in $\mathfrak{g}^{\prime}$ which restricts to $\gamma$. In terms of the obvious notation for standard coordinates, 


\section{P. E. TRAPA}

if $\alpha=e_{i}-e_{i+1}($ with $1 \leqslant i \leqslant n-1)$, then $\alpha_{1}^{\prime}=e_{i}^{\prime}-e_{i+1}^{\prime}$ and $\alpha_{2}^{\prime}=e_{2 n-i}^{\prime}-e_{2 n+1-i}^{\prime}$; and if $\gamma=2 e_{i}$, then $\gamma^{\prime}=e_{i}^{\prime}-e_{2 n+1-i}^{\prime}$.

Fix a system of positive roots for $\mathfrak{h}^{\prime}$ in $\mathfrak{g}^{\prime}$. This restricts to a system of positive roots for $\mathfrak{h}$ in $\mathfrak{g}$. Suppose $\alpha$ is a short simple root for $\mathfrak{h}$ in $\mathfrak{g}$. We want to investigate the conditions under which

$$
\alpha \in \tau(X(Q)) \text { if and only if } \alpha_{1}^{\prime}, \alpha_{2}^{\prime} \in \tau\left(X\left(Q^{\prime}\right)\right) .
$$

Using the explicit calculation of $Q \mapsto Q^{\prime}$, the calculations of $\tau$-invariants for $\mathrm{U}(p, q)$ in [Gar93b, $\S 1.13]$, and the analogous calculations for $\operatorname{Sp}(2 n, \mathbb{R})$, it is easy to see this is always the case except in one family of cases. It can happen that $\alpha$ is a noncompact imaginary root for $X(Q)$ (and hence not in the $\tau$-invariant of $X(Q)$ ), while both $\alpha_{i}^{\prime}$ are complex roots for $X\left(Q^{\prime}\right)$ with $\theta^{\prime}\left(\alpha_{i}^{\prime}\right)<0$ (and so they are both in the $\tau$ invariant of $X\left(Q^{\prime}\right)$ ). As a matter of terminology (for the purposes of this proof), we say that $\alpha$ is an exceptional noncompact imaginary root. The first case where such a root appears is in the rank-two group $\operatorname{Sp}(4, \mathbb{R})$ with $Q$ the unique orbit attached to the Cartan subgroups attached to $\mathbb{C}^{\times}$which does not support a derived functor module. (This orbit has complex dimension three.) Then the short simple root is exceptional noncompact. In this case $\mu(Q)$ is the unique nilpotent orbit corresponding to the partition $4=2+2$ which is not relevant. It may happen, however, in higher rank examples that we may encounter an exceptional noncompact imaginary root $\alpha$ for $X(Q)$ while $\mu(Q)$ is still relevant. However, the following always holds: if $\alpha$ is a simple exceptional noncompact root for $X(Q)$ and $\beta$ is a simple root adjacent to $\alpha$ such that $\beta \in \tau(X(Q))$, then $\mu(Q)$ is not relevant. To prove this, one needs to make a detailed analysis of the combinatorial algorithm computing $\mu(Q)$ given in [Tra05a, §3]. We omit this analysis. The conclusion is that if $\mathcal{O}_{K}$ is relevant, $Q \in \mu^{-1}\left(\mathcal{O}_{K}\right)$, and $\alpha$ is short, then (5.38) holds:

(i) whenever $\alpha$ is not exceptional noncompact imaginary; and

(ii) whenever $\alpha$ is exceptional noncompact imaginary so that there is a short adjacent simple root $\beta \in \tau(X(Q))$.

Now suppose $\gamma$ is a long simple root. A similar analysis as discussed in the previous paragraph allows one to conclude that

$$
\gamma \in \tau(X(Q)) \text { if and only if } \gamma^{\prime} \in \tau\left(X\left(Q^{\prime}\right)\right),
$$

with no additional restrictions on $Q$.

The previous two paragraphs imply that if $Q \in \mu^{-1}\left(\mathcal{O}_{K}\right)$ with $\mathcal{O}_{K}$ relevant, then $X(Q)$ is in the domain of an operator of the form $T_{\alpha \beta}$ with $\alpha$ and $\beta$ short if and only if $X\left(Q^{\prime}\right)$ is in the domain of the operators $T_{\alpha_{1}^{\prime}, \beta_{1}^{\prime}}$ and $T_{\alpha_{2}^{\prime}, \beta_{2}^{\prime}}$. Meanwhile, $X(Q)$ is in the domain of $T_{\alpha \gamma}$ with $\gamma$ long if and only if $X\left(Q^{\prime}\right)$ is in the domain of each $T_{\alpha_{i}^{\prime} \gamma^{\prime}}$. Fix an orbit $Q \in \mu^{-1}\left(\mathcal{O}_{K}\right)$ so that $X(Q)$ satisfies condition (ii) of Hypothesis $(\star)$. Suppose $\alpha$ and $\beta$ are two short simple roots for $\mathfrak{h}$ in $\mathfrak{g}$ that together span a root system of type $\mathrm{A}_{2}$. Fix $Q \in K \backslash \mathfrak{B}$ and suppose $X(Q)$ is in the domain of $T_{\alpha \beta}$. Using the computations in the proof of [Vog79b, Theorem 3.10] distilled in [Gar93b, Definition 1.14.1], and the fact that the relevant hypothesis rules out the exception remarked upon above, one deduces that there exists an orbit $Q_{1}$ so that

$$
T_{\alpha \beta}(X(Q))=X\left(Q_{1}\right) .
$$

Recall that the previous paragraph implies that $X\left(Q^{\prime}\right)$ is in the domain of $T_{\alpha_{1}^{\prime}, \beta_{1}^{\prime}}$ and $T_{\alpha_{2}^{\prime}, \beta_{2}^{\prime}}$; since the roots involved in the two sets of operators are orthogonal, we can consider the composition of the two applied to $X\left(Q^{\prime}\right)$. (The result is independent of the order in which the composition is taken.) Again using the computation in [Vog79b, Theorem 3.10], and the explicit computation of the map $Q \mapsto Q^{\prime}$, we deduce

$$
X\left(Q_{1}^{\prime}\right)=T_{\alpha_{1}^{\prime}, \beta_{1}^{\prime}} \circ T_{\alpha_{2}^{\prime}, \beta_{2}^{\prime}}\left(X\left(Q^{\prime}\right)\right) .
$$




\section{LEADING-TERM CYCLES OF HARISH-CHANDRA MODULES}

It now follows from (5.37) and Theorem 5.1 (for $\mathrm{U}(n, n)$ ) that indeed $Q_{1} \in \mu^{-1}\left(\mathcal{O}_{K}\right)$. Hence we have verified (5.36) whenever $\mathbf{T}$ is a single operator $T_{\alpha \beta}$ with $\alpha$ and $\beta$ short (and $I_{\alpha \beta}(Q)$ necessarily consists of one element).

We now turn to verifying (5.36) for an unequal-length operator. So let $\alpha$ be a short simple root and let $\gamma$ be a long simple root so that together they span a root system of Type $\mathrm{C}_{2}$. Fix $Q \in$ $\mu^{-1}\left(\mathcal{O}_{K}\right)$ and, as usual, assume $\mathcal{O}_{K}$ is relevant. It transpires that the relevant hypothesis rules out the case that $\alpha$ is exceptional noncompact imaginary (in the terminology introduced above). This follows by exactly the same kind of detailed analysis of the combinatorial algorithm computing $\mu(Q)$ given in [Tra05a, $\S 3]$ that we needed above. We omit further details.

Assume now that $X(Q)$ is in the domain of $T_{\alpha \gamma}$. Let $S_{1}$ denote $T_{\alpha \gamma}(X(Q))$. This set has one or two elements, each of which is in the domain of $T_{\alpha \gamma}$. Let $S_{2}$ be the set obtained by applying $T_{\alpha \gamma}$ to each element of $S_{1}$; again $S_{2}$ has one or two elements, and $X(Q)$ is always contained in $S_{2}$. Let $S=S_{1} \cup S_{2}$. (More abstractly, $S$ is the smallest subset of Harish-Chandra modules with trivial infinitesimal character containing $X(Q)$ consisting of modules in the domain of $T_{\alpha \gamma}$ that is actually closed under application of $T_{\alpha \gamma}$.) A relatively short case-by-case analysis shows that $S$ always has three elements and, moreover, since we have assumed that $\alpha$ is not exceptional noncompact imaginary, each element of $S$ is of the form $X\left(Q_{\circ}\right)$ for some orbit $Q_{\circ}$. Hence we may write

$$
S=\left\{X\left(Q_{\mathrm{i}}\right), X\left(Q_{\mathrm{ii}}\right), X\left(Q_{\mathrm{iii}}\right)\right\} .
$$

The case-by-case analysis mentioned above in fact implies that we may arrange the notation so that $T_{\alpha \gamma}\left(X\left(Q_{\mathrm{i}}\right)\right)=X\left(Q_{\mathrm{ii}}\right), T_{\alpha \gamma}\left(X\left(Q_{\mathrm{iii}}\right)\right)=X\left(Q_{\mathrm{ii}}\right)$, and

$$
T_{\alpha \gamma}\left(X\left(Q_{\mathrm{ii}}\right)\right)=\left\{X\left(Q_{\mathrm{i}}\right), X\left(Q_{\mathrm{iii}}\right)\right\} .
$$

We next implement (to the greatest possible extent) the technique used to treat the equal-length operator situation. A case-by-case analysis shows that the technique always applies to show that either

$$
\mu\left(Q_{\mathrm{ii}}\right)=\mu\left(Q_{\mathrm{i}}\right) \quad \text { or } \quad \mu\left(Q_{\mathrm{ii}}\right)=\mu\left(Q_{\mathrm{iii}}\right) .
$$

More precisely, we can show that there is a well-defined composition, say $\mathbf{T}^{\prime}$, of equal-length operators for $\mathrm{U}(n, n)$ so that either

$$
\mathbf{T}^{\prime}\left(X\left(Q_{\mathrm{ii}}^{\prime}\right)\right)=X\left(Q_{\mathrm{i}}^{\prime}\right) \quad \text { or } \quad \mathbf{T}^{\prime}\left(X\left(Q_{\mathrm{ii}}^{\prime}\right)\right)=X\left(Q_{\mathrm{iii}}^{\prime}\right) .
$$

Arguing as above, this implies (5.40). After further relabeling we now assume the first case holds, namely

$$
\mu\left(Q_{\mathrm{ii}}\right)=\mu\left(Q_{\mathrm{i}}\right) .
$$

Recall that we are assuming that $X(Q)$ satisfies Hypothesis $(\star)$ for the orbit $Q \in \mu^{-1}\left(\mathcal{O}_{K}\right)$ and we are trying to establish (5.36). There are three cases to consider: $Q=Q_{\mathrm{i}}, Q=Q_{\mathrm{ii}}$, or $Q=Q_{\mathrm{iii}}$. In all cases, we now argue that (5.36) is ultimately reduced to proving

$$
\text { if } \operatorname{Ann}\left(X\left(Q_{\mathrm{i}}\right)\right) \neq \operatorname{Ann}\left(X\left(Q_{\mathrm{iii}}\right)\right) \text {, then } \mu\left(Q_{\mathrm{i}}\right)=\mu\left(Q_{\mathrm{iii}}\right) \text {. }
$$

To see this, first assume $Q=Q_{\mathrm{i}}$. Then (5.41) says that (5.36) holds whenever $\operatorname{Ann}\left(X\left(Q_{\mathrm{i}}\right)\right)=$ $\operatorname{Ann}\left(X\left(Q_{\mathrm{iii}}\right)\right)$; hence we are reduced to (5.42). Next assume $Q=Q_{\mathrm{ii}}$. Then (5.41) again says that (5.36) holds whenever $\operatorname{Ann}\left(X\left(Q_{\mathrm{i}}\right)\right)=\operatorname{Ann}\left(X\left(Q_{\text {iii }}\right)\right)$; hence we are again reduced to (5.42). Finally assume $Q=Q_{\mathrm{iii}}$. If $\mu\left(Q_{\mathrm{i}}\right) \neq \mu\left(Q_{\mathrm{iii}}\right)$, a case-by-case analysis with the algorithm of [Tra05a, $\S 3]$ shows that $\mu\left(Q_{\text {iii }}\right) \subsetneq \mu\left(Q_{\mathrm{i}}\right)$. Hence the GK-dimension of $X\left(Q_{\mathrm{i}}\right)$ (which is weakly greater than the dimension of $\left.\mu\left(Q_{\mathrm{i}}\right)\right)$ is strictly greater than the dimension of $\mu\left(Q_{\mathrm{iii}}\right)$ which, by the assumption that $Q=Q_{\text {iii }}$ satisfies condition (ii) of Hypothesis $(\star)$, is the GK-dimension of $X\left(Q_{\text {iii }}\right)$. Hence the GK-dimension of $X\left(Q_{\mathrm{i}}\right)$ is strictly greater than that of $X\left(Q_{\mathrm{iii}}\right)$. However, this contradicts the fact 


\section{P. E. TRAPA}

that $X\left(Q_{\mathrm{i}}\right)$ can be obtained from applying the (GK-dimension preserving) operator $T_{\alpha \gamma}$ successively to $X\left(Q_{\text {iii }}\right)$. This contradiction shows that if $Q=Q_{\text {iii }}$, then indeed $\mu\left(Q_{\mathrm{i}}\right)=\mu\left(Q_{\mathrm{iii}}\right)$ and (5.36) holds automatically. Hence, we really are reduced to (5.42) and we may assume $Q=Q_{\mathrm{i}}$ or $Q_{\mathrm{ii}}$. By (5.41), part (ii) of Hypothesis $(\star)$ holds for $Q_{\mathrm{i}}$ if and only if it holds for $Q_{\mathrm{ii}}$. So indeed we may assume it holds for both of them.

Retain the setting of (5.42) and assume that $Q=Q_{\text {ii }}$. Recall (as mentioned in $\S 2.2$ ) that primitive ideals with trivial infinitesimal character in Type $\mathrm{C}$ are in bijection to standard domino tableaux with special shape. Moreover, there is an action of $T_{\alpha} \gamma$ on domino tableaux satisfying the appropriate condition compatible with this bijection [Gar90, Gar92, Gar93a]. Then the domino tableaux attached by [Tra05a, $\S 3$ ] to $Q_{\mathrm{i}}$ and $Q_{\mathrm{iii}}$, which parametrize the annihilators of $X\left(Q_{\mathrm{i}}\right)$ and $X\left(Q_{\mathrm{iii}}\right)$, differ by moving through a single closed cycle in the sense of [Gar90, Gar92, Gar93a]. The construction of [Tra05a, §3] then guarantees that $Q_{\mathrm{i}}$ and $Q_{\mathrm{iii}}$ lie in the same fiber of $\mu$, as desired.

Theorem 5.43 (McGovern). The conclusion of Theorem 5.32 holds for the orthogonal groups $\mathrm{SO}(p, q)$ with $p+q$ even.

Sketch of the proof. The argument leading to Theorem 5.32 applies in Type D with appropriate (and sometimes rather intricate) modifications. One needs to replace the unequal-length operators $T_{\alpha \gamma}$ appearing in the proof of Theorem 5.32 with the more complicated ones $T_{D}$ and $S_{D}$ defined in [McG98a] using the main results in [GV92]. By making further explicit calculations, one rules out a longer list of case-by-case exceptions that arise. We omit the details.

Remark 5.44. For $\mathrm{Sp}(2 n, \mathbb{R})$ and $\mathrm{SO}(p, q)$, the proofs of Theorems 5.32 and 5.43 provide an effective way to compute the annihilator (and associated variety) of each Harish-Chandra module of the form $X(Q)$ for $Q \in \mu^{-1}\left(\mathcal{O}_{K}\right)$ and $\mathcal{O}_{K}$ relevant. As W. McGovern has pointed out in unpublished work, this provides the as-of-yet most complete computation of annihilators and associated varieties for these groups. Because the unitary group $\mathrm{U}(n, n)$ (all of whose Cartan subgroups are connected) is used in the proofs, one cannot conclude much about computing annihilators of modules of the form $X(Q, \psi)$ with $\psi$ nontrivial. Such nontrivial local systems present serious complications, and the problem of computing annihilators for all representations of $\operatorname{Sp}(2 n, \mathbb{R})$ and $\mathrm{SO}(p, q)$ remains open. (D. Garfinkle, in personal communication to the author, has suggested that she has made progress on this problem.)

Remark 5.45. Since Theorems 5.32 and 5.43 verify Hypothesis $(\star)$ for all complex special orbits in Types B, C, and D, it may seem that Theorem 5.2 is superfluous, but this in not the case. For the kinds of complex orbits that appear in Theorem 5.2 (see Remark 5.25), the ordering giving the triangularity of the matrix in Theorem 3.20 provided by using $\operatorname{Sp}(p, q)$ or $\mathrm{SO}^{*}(2 n)$ can be wildly different from the ordering provided by using $\mathrm{Sp}(2 n, \mathbb{R})$ or $\mathrm{SO}(p, q)$. The orderings, which originate in the closure order for the orbits of two different reductive groups on the same flag variety, in fact bear no resemblance to each other whatsoever. This is a somewhat mysterious feature of our results.

Remark 5.46. For $\mathrm{G}_{2}$ and the complex orbits relevant for the rank one form of $\mathrm{F}_{4}$, the hypothesis is easy to verify. We omit the details.

Remark 5.47. The main results of [ABV92], especially those of ch. 26, provide an algorithm to define a set of Arthur packets whose union conjecturally exhausts the automorphic spectrum of a group $G_{\mathbb{R}}$ arising as the real points of a connected reductive algebraic group defined over $\mathbb{R}$. (The automorphic spectrum consists of those (unitary) representations that appear in $L^{2}\left(\Gamma \backslash G_{\mathbb{R}}\right.$ ) for a congruence subgroup $\Gamma$ of $G_{\mathbb{R}}$.) The algorithm depends on the computation of $\mathcal{C V}(X)$ for Harish-Chandra modules $X$ with trivial infinitesimal character and, as we mentioned in $\S 3$, there 


\section{LEADING-TERM CYCLES OF HARISH-CHANDRA MODULES}

is no known effective way to compute these characteristic cycles. The main results of this paper give restrictions on the leading terms of such cycles. While these results are far from definitive, they lead to highly nontrivial conclusions in examples. Using [ABV92], such conclusions should have applications to computing the smallest representations in an Arthur packet. (Because the duality of [Vog82] is involved in the definition of such packets, the computation of leading-term cycles (the 'largest' piece of the characteristic cycle) corresponds to information about the smallest representations in an Arthur packet. For many interesting packets (such as unipotent packets) all representations have the same size.) Remark 3.14 is particularly intriguing since it suggests a potential relationship between the computation of Arthur packets for different real forms of the same complex group. It would be interesting to make this more explicit.

\section{ACKNOWLEDGEMENTS}

I thank W. McGovern for numerous helpful conversations as well as continued encouragement during the course of this project. His paper [McG00] was the inspiration for the current paper. I thank him for allowing me to include Theorem 5.43 here. I also thank D. Miličić for helpful conversations related to [Cha93].

\section{REFERENCES}

ABV92 J. Adams, D. Barbasch and D. A. Vogan Jr, The Langlands classification and irreducible characters for real reductive groups, Progress in Mathematics, vol. 104 (Birkhäuser, Boston, MA, 1992).

BV82 D. Barbasch and D. A. Vogan, Primitive ideals and orbital integrals in complex classical groups, Math. Ann. 259 (1982), 153-199.

BV83 D. Barbasch and D. A. Vogan, Primitive ideals and orbital integrals in complex exceptional groups, J. Algebra 80 (1983), 350-382.

BB82 W. Borho and J.-L. Brylinski, Differential operators on homogeneous spaces I. Irreducibility of the associated variety for annihilators of induced modules, Invent. Math. 69 (1982), 437-476.

BB85 W. Borho and J.-L. Brylinski, Differential operators on homogeneous spaces III. Characteristic varieties of Harish-Chandra modules and of primitive ideals, Invent. Math. 80 (1985), 1-68.

Cha93 J.-T. Chang, Asymptotics and characteristic cycles for representations of complex groups, Compositio Math. 88 (1993), 265-283.

CM93 D. Collingwood and W. M. McGovern, Nilpotent orbits in semisimple Lie algebras (Van Nostrand Reinhold, New York, 1993).

Gar90 D. Garfinkle, On the classification of primitive ideals for complex classical Lie algebras I, Compositio Math. 75 (1990), 135-169.

Gar92 D. Garfinkle, On the classification of primitive ideals for complex classical Lie algebra II, Compositio Math. 81 (1992), 307-336.

Gar93a D. Garfinkle, On the classification of primitive ideals for complex classical Lie algebras III, Compositio Math. 88 (1993), 187-234.

Gar93b D. Garfinkle, The annihilators of irreducible Harish-Chandra modules for $\mathrm{SU}(p, q)$ and other type $A_{n-1}$ groups, Amer. J. Math. 115 (1993), 305-369.

GV92 D. Garfinkle and D. A. Vogan, On the structure of Kazhdan-Lusztig cells for branched Dynkin diagrams, J. Algebra 153 (1992), 91-120.

Hot84 R. Hotta, On Joseph's construction of Weyl group representations, Tohoku Math. J. (2) 36 (1984), 49-74.

Jos77 A. Joseph, A characteristic variety for the primitive spectrum of a semisimple Lie algebra, in Non-commutative harmonic analysis, Actes Colloque, Marseille-Luminy, 1976, Lecture Notes in Mathematics, vol. 587 (Springer, Berlin, 1977), 102-118. 


\section{LEADING-TERM CYCLES OF HARISH-CHANDRA MODULES}

Jos80a A. Joseph, Goldie rank in the enveloping algebra of a semisimple Lie algebra. I, J. Algebra 65 (1980), 269-283.

Jos80b A. Joseph, Goldie rank in the enveloping algebra of a semisimple Lie algebra. II, J. Algebra 65 (1980), 284-306.

Jos84 A. Joseph, On the variety of a highest weight module, J. Algebra 88 (1984), 238-278.

Jos89 A. Joseph, On the characteristic polynomials of orbital varieties, Ann. Sci. École Norm. Sup. (4) 22 (1989), 569-603.

KV95 A. W. Knapp and D. A. Vogan Jr, Cohomological induction and unitary representations, Princeton Mathematical Series, vol. 45 (Princeton University Press, Princeton, NJ, 1995).

KR71 B. Kostant and S. Rallis, Orbits and representations associated with symmetric spaces, Amer. J. Math. 93 (1971), 753-809.

McG98a W. M. McGovern, Left cells and domino tableaux in classical Weyl groups, Compositio Math. 101 (1998), 77-98.

McG98b W. M. McGovern, Cells of Harish-Chandra modules for real classical groups, Amer. J. Math. 120 (1998), 211-228.

McG00 W. M. McGovern, A triangularity result for associated varieties of highest weight modules, Comm. Algebra 28 (2000), 1835-1843.

Mel93 A. Melnikov, Irreducibility of the associated varieties of simple highest weight modules in $\mathfrak{s l}(n)$, C. R. Acad. Sci. Paris Sér. I Math. 316 (1993), 53-57.

Mil93 D. Miličić, Localization and representation theory of reductive Lie groups, Preprint (1993), http://www.math.utah.edu/ milicic/.

Ros91 W. Rossmann, Invariant eigendistributions on a semisimple Lie algebra and homology classes on the conormal variety II: Representations of Weyl groups, J. Funct. Anal. 96 (1991), 155-193.

Spa77 N. Spaltenstein, On the fixed point set of a unipotent element on the variety of Borel subgroups, Topology 16 (1977), 203-204.

Ste88 R. Steinberg, An occurrence of the Robinson-Schensted correspondence, J. Algebra 113 (1988), $523-528$.

Tan88 T. Tanisaki, Characteristic varieties of highest weight modules and primitive quotients, in Representations of Lie groups, Kyoto, Hiroshima, 1986, Advanced Studies in Pure Mathematics, vol. 14 (Academic Press, Boston, MA, 1988), 1-30.

Tra99 P. E. Trapa, Generalized Robinson-Schensted algorithms for real Lie groups, Internat. Math. Res. Notices 15 (1999), 803-834.

Tra05a P. E. Trapa, Symplectic and orthogonal Robinson-Schensted algorithms, J. Algebra 286 (2005), 386-404.

Tra05b P. E. Trapa, Richardson orbits for real groups, J. Algebra 286 (2005), 361-385.

Vog78 D. A. Vogan, Gelfand-Kirillov dimension for Harish-Chandra modules, Invent. Math. 48 (1978), 75-98.

Vog79a D. A. Vogan, A generalized $\tau$-invariant for the primitive spectrum of a semisimple Lie algebra, Math. Ann. 242 (1979), 209-224.

Vog79b D. A. Vogan, Irreducible characters of semisimple Lie groups I, Duke Math. J. 46 (1979), 61-108.

Vog82 D. A. Vogan, Irreducible characters of semisimple Lie groups IV. Character-multiplicity duality, Duke Math. J. 49 (1982), 943-1073.

Vog91 D. A. Vogan, Associated varieties and unipotent representations, in Harmonic analysis on reductive groups, Brunswick, ME, 1989, Progress in Mathematics, vol. 101 (Birkhäuser, Boston, MA, 1991), 315-388.

VZ84 D. A. Vogan and G. Zuckerman, Unitary representations with nonzero cohomology, Compositio Math. 53 (1984), 51-90.

Peter E. Trapa ptrapa@math.utah.edu

Department of Mathematics, University of Utah, Salt Lake City, UT 84112, USA 